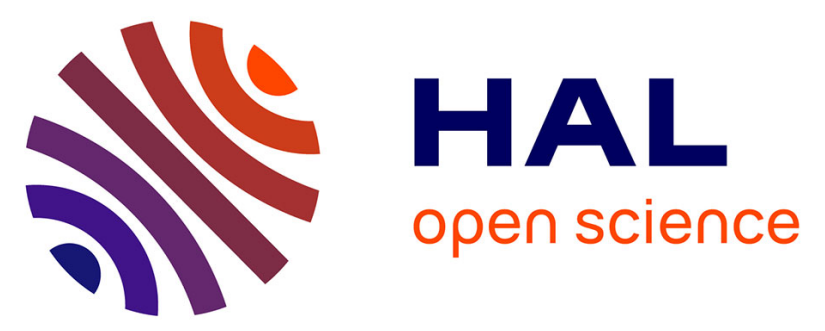

\title{
PELICAN: Panoramic millimeter-wave radar for perception in mobile robotics applications. Part 1: Principles of FMCW radar and of 2D image construction
}

R. Rouveure, P. Faure, M.O. Monod

\section{- To cite this version:}

R. Rouveure, P. Faure, M.O. Monod. PELICAN: Panoramic millimeter-wave radar for perception in mobile robotics applications. Part 1: Principles of FMCW radar and of 2D image construction. Robotics and Autonomous Systems, 2016, 81, pp.1-16. 10.1016/j.robot.2016.04.001 . hal-01383402

\author{
HAL Id: hal-01383402 \\ https://hal.science/hal-01383402
}

Submitted on 18 Oct 2016

HAL is a multi-disciplinary open access archive for the deposit and dissemination of scientific research documents, whether they are published or not. The documents may come from teaching and research institutions in France or abroad, or from public or private research centers.
L'archive ouverte pluridisciplinaire HAL, est destinée au dépôt et à la diffusion de documents scientifiques de niveau recherche, publiés ou non, émanant des établissements d'enseignement et de recherche français ou étrangers, des laboratoires publics ou privés. 


\title{
PELICAN: Panoramic Millimeter-Wave Radar for Perception in Mobile Robotics Applications \\ Part 1: Principles of FMCW Radar and of 2D Image Construction
}

\author{
Raphaël Rouveure, Patrice Faure and Marie-Odile Monod
}

National Research Institute of Science and Technology for Environment and Agriculture (IRSTEA), Technologies and Information Systems Research Unit (TSCF), Clermont-Ferrand Regional Center, 9 avenue Blaise Pascal, CS 20085, 63178 Aubière, France

Email addresses: raphael.rouveure@irstea.fr; patrice.faure@irstea.fr; marie-odile.monod@irstea.fr

Correspondence should be addressed to Raphaël Rouveure, raphael.rouveure@irstea.fr

\begin{abstract}
Robust environmental perception is a crucial parameter for the development of autonomous ground vehicle applications, especially in the field of agricultural robotics which is one of the priorities for the Horizon 2020 robotics funding (EU funding program for research and innovation). Because of uncontrolled and changing environmental conditions in outdoor and natural environments, data from optical sensors classically used in mobile robotics can be compromised and unusable. In such situations, millimeter-wave radar can provide an alternative and complementary solution for perception tasks. The aim of this paper is to present the PELICAN radar, a millimeter-wave radar specifically designed for mobile robotics applications, including obstacle detection, mapping and situational awareness in general. In this first of a two-part paper, the choice of a frequency-modulated continuous-wave radar is explained and the theoretical elements of this solution are detailed. PELICAN radar is using a rotating fan-beam antenna, and the construction of $2 \mathrm{D}$ representations of the surrounding environments with radar data is described through simulation results. The second part of the paper will be devoted for a detailed description of PELICAN radar, as well as experimental results.
\end{abstract}

\section{Keywords}

MMW radar; FMCW radar; mobile robotics; 2D imaging; robust perception

\section{Introduction}

Robust perception is still a sensitive and challenging process in outdoor environments for Autonomous Ground Vehicles (AGV). Robots have to deal with complex and changing situations, including variations of ambient light level, day/night cycles, weather conditions (fog, rain, snow) or presence of obscurants (dust, smoke). In such situations, sometimes called Degraded Visual Environments (DVE), optical sensors (vision, laser) classically used in mobile robotics applications can be partially or completely ineffective, and the mission of the robot will not be completed.

It is no longer necessary to demonstrate the robustness and the efficiency of Millimeter-Wave (MMW) radars for perception tasks in outdoor environments. Because of a millimeter wavelength, MMW radars provide robust information even in degraded visual conditions. Such capabilities are extensively used for example in the military domain (air and maritime surveillance, missile guidance, etc.), in the field of civil aviation (approach radar, surface movement radar) or in the field of remote sensing (observation of Earth and other planets of the solar system). For the development of AGV applications, radar technology must be progressively adapted for smaller platforms, in terms of dimension, weight, energy consumption and cost. 
The agricultural domain is a promising sector for AGV applications [1],[2] and manufacturers are already working on the development of industrial solutions [3],[4]. But the autonomy of these systems can be limited when they are faced with environmental conditions that cannot be controlled in outdoor and natural environments, and that are often harsh for perception sensors. Irstea Institute in Clermont-Ferrand (France) is involved in several research projects for the development of robotics solutions for the agricultural domain ${ }^{1}$, and it has naturally taken an interest in the development of a robust perception system based on MMW radar ${ }^{2}$.

Numerous research teams have used and are using MMW radars within the framework of mobile robotics domain. Obstacle detection is widely studied in automotive applications, for the detection of moving and static targets (cars, pedestrians) [5],[6],[7] or for the estimation of road geometry [8]. The imaging capabilities of radars are also used for visualization and characterization of the environment [9],[10],[11],[12], 2D/3D mapping and simultaneous localization and mapping (SLAM) applications [13],[14],[15]. Several researches are also involved in radar-vision fusion for obstacle detection and outdoor reconstruction [16],[17],[18],[19] relying on the complementarity of the sensors: robustness to environmental conditions and depth detection ability of the radar; high spatial resolution of the vision. One problem for the development of radar-based solutions in mobile robotics is the radar itself, because the number of radars available on the market is not so important. In the automotive domain, several specific radars are under development for vehicle and obstacle detection in degraded atmospheric conditions; but their use outside the field for which they were originally designed can be difficult due to specific design considerations. A few commercial systems can be found on the market such as a $77 \mathrm{GHz}$ radar from Navtech Radar Ltd Company, but these radars have not been specifically developed for AGV applications. Our objective was to develop a specific MMW radar for perception in mobile robotics applications, with the ambition of making this radar available for the robotics community, through the availability of databases, collaboration on research programs and perhaps in the future the marketing of the radar.

The aim of this paper is to present PELICAN radar (see Figure 1). PELICAN is a panoramic radar developed at Irstea Institute for perception in mobile robotics applications. It is a low-power K-band radar $(24 \mathrm{GHz})$, and it is based on a frequency-modulated continuous-wave emission. The radar is using a fan-beam antenna, which allows to build $2 \mathrm{D}$ representations of the surrounding environment. Lightweight (5 kg) and small sized (diameter $40 \mathrm{~cm}$, height $23 \mathrm{~cm}$ ), PELICAN can be easily positioned on various mobile platforms.

In this first part, the theoretical elements of the developed radar are presented, as well as simulation results. The second part of the paper will be devoted for a detailed description of PELICAN radar, and for experimental results. The principle of frequency modulation used for the radar is presented in section 2, with a focus on the problem of simultaneous range and velocity measurement. Section 3 develops the criteria that have to be taken into account for the choice of the carrier frequency of the radar, such as radar dimensions or regulation constraints. The adopted solution to build 2D images of the environment with radar positioned on a robot is described in section 4 , and it is illustrated with simulation results. And section 5 concludes the paper.

\footnotetext{
Examples of research projects led by Irstea Institute:

1 - PUMAgri: Universal mobile platform for agriculture

- ActiSurTT: Active devices for vehicles security in off-road environments

- SafePlatoon: Security of convoy of autonomous vehicles.

2 - PELICAN: Radar Perception Localization and Mapping for Natural Areas.

- QUAD-AV: Ambient Awareness for Autonomous Agricultural Vehicles.
} 


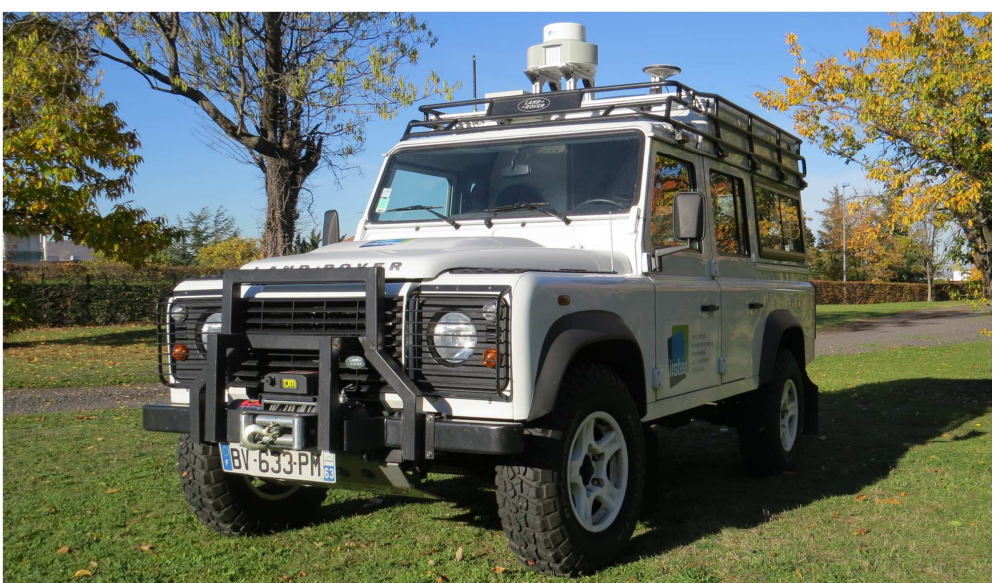

(a)

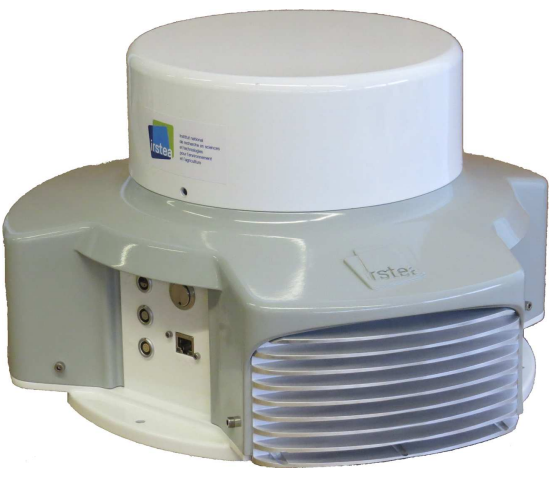

(b)

FIGURE 1: PELICAN radar (a) positioned on an experimental vehicle, and (b) detailed view.

\section{FMCW Radar Principle}

Radars allow the location of objects in space by transmitting electromagnetic energy, and observing the returned echo. In the radar domain, two major families can be used to estimate the position of an object: pulse radars and continuous radars.

Basically, pulse radars transmit a high powered short pulse, after which the receiver is switched on in order to receive the echoes [20],[21]. The presence of one or several echoes indicates the presence of one or several targets. The range $r_{i}$ to target $i$ is estimated through the measurement of the delay time $\tau_{i}$ between pulse transmission and pulse reception, with

$$
r_{i}=\frac{c \tau_{i}}{2}
$$

where $c$ is the light velocity. Pulse radar transmits pulse of duration $\tau_{d .}$ During this transmission duration, the receiver is switched off for protection purposes, and the radar cannot detect any targets: the transmission duration $\tau_{d}$ defines a blind zone from range zero to range $\delta r=c \tau_{d} / 2$. The distance $\delta r$ also defines the range resolution, i.e. the ability of the radar to distinguish two close targets. For autonomous robots applications requiring accurate radar-target distance measurements over short distances, a large value of $\delta r$ can lead to an unacceptable configuration. Thus, a major problem with pulse radars is to be able to operate over short time durations - in order to achieve a high range resolution - a very high peak power signal - in order to have a reliable signal reception. For that reason, frequency modulated continuous wave radars provide competitive solutions for distance measurement in short range applications.

\subsection{FMCW Principle}

Unmodulated continuous wave $(C W)$ radar transmits continuously a constant frequency $f_{0}$ with constant amplitude, and measures the frequency difference between the transmitted and received signals. If the echo signal is reflected on a static target (radial velocity $V_{r}=0$ ), transmitted and received frequency are equal. If the echo signal is reflected on a moving target (radial velocity $V_{r} \neq 0$ ), transmitted and received frequencies are different because the frequency of the reflected signal is shifted by the amount of the Doppler frequency $f_{d}$. 
The radial velocity $V_{r i}$ between the radar and the target $i$ can be estimated using the well-known Doppler shift formula [20],[22]

$$
V_{r i}=\frac{c f_{d}}{2 f_{0}}
$$

As no runtime measurement is required with $\mathrm{CW}$ radar, no distance determination can be made and the distance between the radar and the moving target $i$ cannot be computed. If the distance must be determined, the transmitted signal can be modulated in order to get a time reference of the received echoes: this is the objective of the frequency modulation.

The principle of frequency modulated continuous wave (FMCW) radar is known and used for several decades [20],[23]. In FMCW radars, the oscillator generates a signal of linearly increasing frequency $\Delta f$ over a period $t_{m}$. This signal is transmitted into the air via the antenna. At the receiver stage, a part of the transmitted signal is mixed with the signals received from the $i$ targets present in the field of view of the radar. The signal which appears at the output of the mixer is filtered and amplified in order to isolate the beat signal $s_{b}$.

Let us consider $i$ targets located at distance $r_{i}$ from the radar. We assume to have a static configuration, i.e. stationary radar and no moving targets. The transmitted signal is linearly modulated over a period $t_{m}=1 / f_{m}$ with a sawtooth function, with a sweep frequency $\Delta f$ centered about $f_{0}$ (see Figure 2). In that case, the beat signal $s_{b}$ can be written as [20],[23],[24]

$$
s_{b}(t)=k \sum_{i} a_{t} a_{r i} \cos (2 \pi \underbrace{\left(2 \Delta f f_{m} \frac{r_{i}}{c}\right)}_{f_{b i}} t+\Phi_{i}),
$$

where $a_{t}$ is the amplitude of transmitted signal, $a_{r i}$ and $\Phi_{i}$ respectively the amplitude and a phase term of the signal received from target $i$, and $k$ a mixer coefficient.

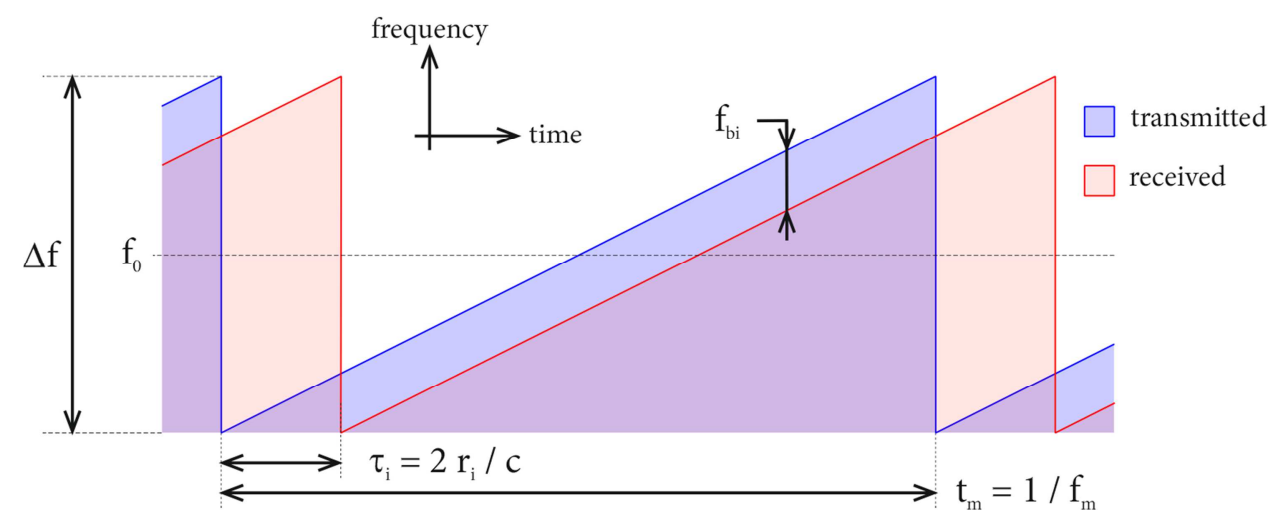

FIGURE 2: FMCW radar principle. The transmitted signal is linearly modulated over a period $t_{m}$ with a sawtooth function. The received signal highlights the same frequency modulation, with a time delay $\tau_{i}$ corresponding to the radar-target distance $r_{i} . f_{0}$ is the carrier frequency, $\Delta f$ the sweep frequency and $f_{m}$ the modulation frequency.

As it can be seen in (3), the beat signal $s_{b}$ is the sum of $i$ frequency components $f_{b b}$, (plus a phase term $\Phi_{i}$ ), each of them corresponding to a particular target $i$

$$
f_{b i}=2 \Delta f f_{m} \frac{r_{i}}{c}
$$


As $\Delta f$ and $f_{m}$ are radar constants, one can see that $f_{b i}$ is proportional to the radar-target distance $r_{i}$, and the measurement of $f_{b i}$ allows determining $r_{i}$

$$
r_{i}=\frac{c f_{b i}}{2 \Delta f f_{m}} .
$$

The maximum range $r_{a m b}$ before ambiguity is given by the period $t_{m}$ of the modulation law

$$
r_{a m b}=\frac{c t_{m}}{2}=\frac{c}{2 f_{m}}
$$

From (3), we see that the amplitudes of the frequency components of the beat signal are proportional to the term $\left(a_{t} a_{r i}\right)$. Considering that $a_{t}$ is constant, the amplitudes of the frequency components are proportional to the amplitudes $a_{r i}$ of the received signals. The radar equation is an efficient tool to study the parameters that affect $a_{r i}$ The radar equation gives a relationship between the expected received power $p_{r}$ from a target, its radar cross section (RCS) $\sigma$, its range $r$, and intrinsic radar characteristics [20]. The simple form of the radar equation is given by

$$
p_{r}=\frac{p_{t} G^{2} \lambda^{2} \sigma}{(4 \pi)^{3} r^{4}}
$$

with $p_{t}$ transmitted power, $\lambda$ wavelength and $G$ antenna gain considering a monostatic case (i.e. the same antenna is used for transition and reception). $p_{t}, G$ and $\lambda$ are constant for a given radar, so $a_{r i}$ only depends on $\sigma$ and $r_{i}$

$$
a_{r i} \propto \frac{\sqrt{\sigma}}{r_{i}^{2}}
$$

If the radar-target distance $r_{i}$ is known, the measurement of $a_{r i}$ allows to estimate $\sigma . \sigma$ is expressed in meter square, and it indicates how a target re-radiates the energy of the incident radar signal. In the case of spatially extended targets (such as ground or sea), the term backscatter coefficient $\sigma_{0}$ is introduced: it is the normalized RCS, i.e. the average RCS per unit of surface.

An example of simulated radar signal based on (3) is shown in Figure 3. The main radar parameters for this simulation are presented in Table 1.

TABLE 1: Characteristics of the simulated FMCW radar.

\begin{tabular}{ll}
\hline Transmitted power $p_{t}$ & $20 \mathrm{dBm}$ \\
Antenna gain G & $100(20 \mathrm{~dB})$ \\
Carrier frequency $f_{0}$ & $24 \mathrm{GHz}$ \\
Sweep frequency $\Delta f$ & $250 \mathrm{MHz}$ \\
Frequency modulation $f_{m}$ & $360 \mathrm{~Hz}$ \\
\hline
\end{tabular}

Two static trihedral metallic corners with an edge dimension of $8 \mathrm{~cm}$ and $20 \mathrm{~cm}$ (respectively) are located at range $30 \mathrm{~m}$ and $40 \mathrm{~m}$ (respectively). Trihedral metallic corners are canonical targets commonly used in radar domain for calibration, and for which the RCS $\sigma_{\text {trihedral }}$ can be calculated analytically. The peak RCS of such a trihedral is given by [25]

$$
\sigma_{\text {trihedral }}=\frac{4 \pi a^{4}}{3 \lambda^{2}}
$$


where $a$ is the edge dimension of the trihedral corner, assuming that $a$ is large with respect to the wavelength $\lambda$. The simulated beat signal $s_{b}$ is shown in Figure 3(a), considering a signal-to-noise ratio (SNR) of $10 \mathrm{~dB}$. The radar spectrum (Figure 3(b)) is computed with a 1024-point FFT: it highlights two main peaks, corresponding to the trihedral corners. The amplitude difference of $10.9 \mathrm{dBm}$ is introduced by the RCS of the targets and the radar-target distances.

(a)

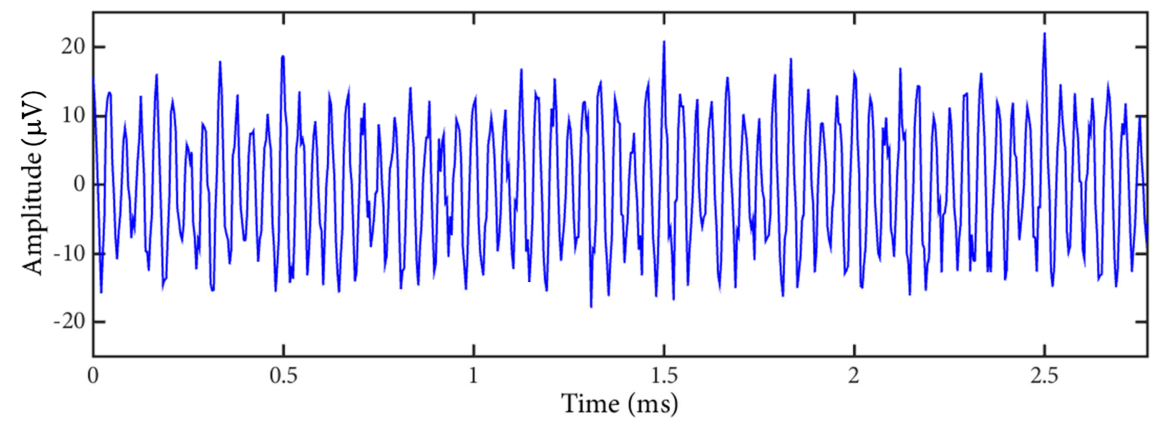

(b)

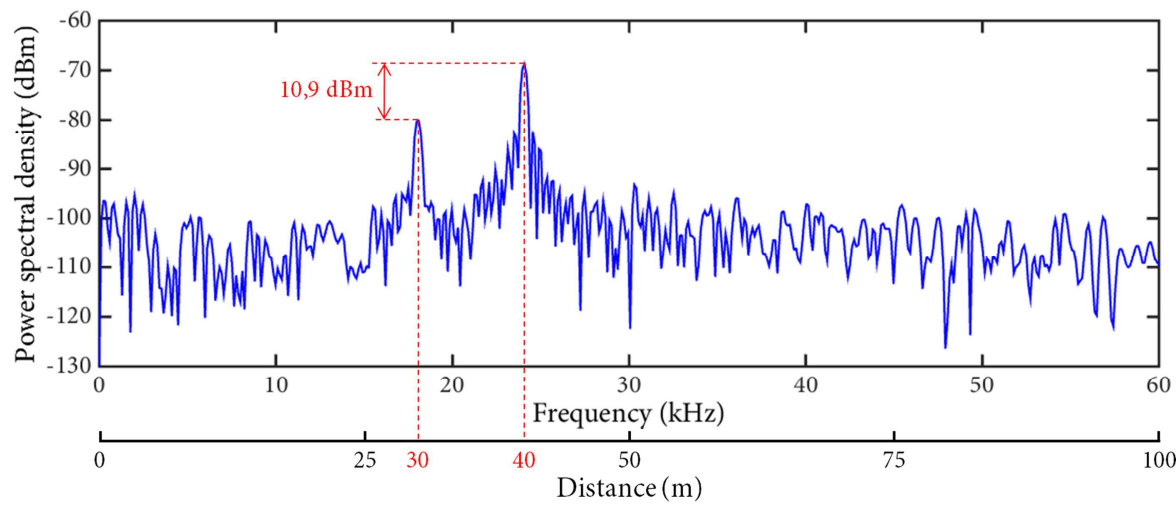

FIGURE 3: Example of simulated FMCW radar signal. Two trihedral metallic corners are located at range $30 \mathrm{~m}$ and $40 \mathrm{~m}$, with RSC equal to $1.1 \mathrm{~m} 2(0.4 \mathrm{dBsm})$ and $42.9 \mathrm{~m} 2(16.3 \mathrm{dBsm})$ respectively. (a) Temporal beat signal $s_{b}$. (b) Radar spectrum computed with a 1024-point FFT.

\subsection{Range Resolution}

From (5), one can see that the radar-target distance $r$ is proportional to the beat frequency $f_{b}$. In that case, it can be assumed that the range resolution is equivalent to a frequency resolution. With a classical FFT frequency analysis, the frequency resolution $\delta f$ is determined by the observation time, i.e. the modulation period $t_{m}$ when considering FMCW radar

$$
\delta f=\frac{1}{t_{m}}=f_{m} .
$$

The increase of $t_{m}$, while maintaining the same sweep frequency $\Delta f$, leads to an increase of the frequency resolution $\delta f$, but the range resolution $\delta r$ is not affected because at the same time the measured distances $r$ are increased (see (5)).

In the case of a FMCW radar, the range resolution $\delta r$ can be estimated from (5), by substituting the beat frequency $f_{b}$ with the frequency resolution $\delta f$

$$
\delta r=\delta f \frac{c}{2 \Delta f f_{m}} .
$$


Substituting (10) in (11), we obtain the well-known relationship between the signal bandwidth and the range resolution

$$
\delta r=\frac{c}{2 \Delta f} .
$$

$\delta r$ defines the distance resolving power, i.e. the ability of the radar to separate (to see as distinct) two targets fairly close together. (12) holds true when considering two reflectors of equal cross section. From (12), one can see that $\delta r$ only depends on the sweep frequency $\Delta f$, so an improvement of the range resolution is obtained with an increase of the sweep frequency. A simulation is presented in Figure 4, considering the signal model (3). Two point targets are located at range $59.8 \mathrm{~m}$ and $60.2 \mathrm{~m}$ in front of the radar. With $\Delta f=200 \mathrm{MHz}$, the theoretical range resolution $\delta d$ is equal to $0.75 \mathrm{~m}$ and the targets are not resolved (Figure 4(a)), even in $t_{m}$ is doubled (Figure 4(b)). In Figure 4(c), it can be seen that the targets are resolved if the sweep frequency is doubled $(\Delta f=400 \mathrm{MHz})$.

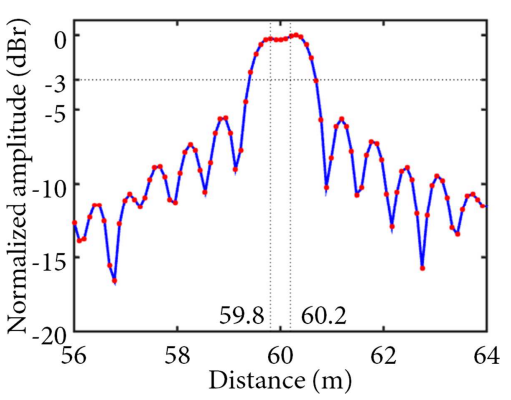

(a)

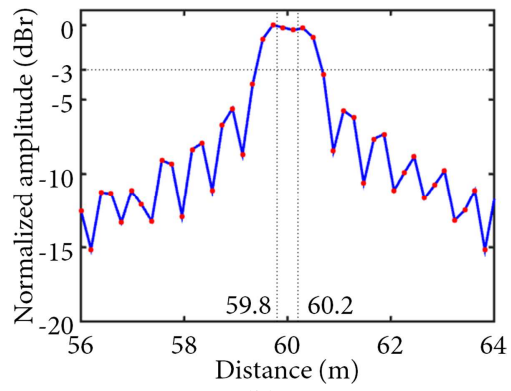

(b)

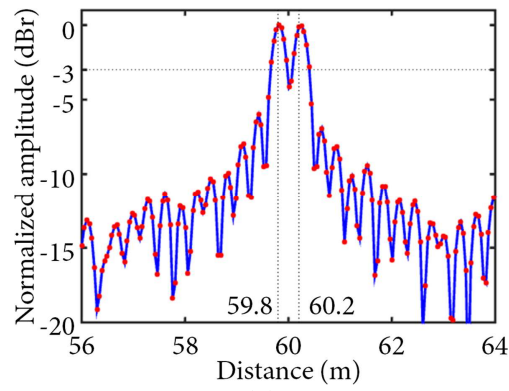

(c)

FIGURE 4: Simulation a FFT spectrum analysis. Two targets are located at ranges $59.8 \mathrm{~m}$ and $60.2 \mathrm{~m}$ in front of the radar. (a) $t_{m}=5.6 \mathrm{~ms}$ and $\Delta f=200 \mathrm{MHz}$. The targets are not resolved. (b) $t_{m}=11.2 \mathrm{~ms}$ and $\Delta f=200 \mathrm{MHz}$. The range resolution is unchanged if $t_{m}$ is increased. (c) $t_{m}=5.6 \mathrm{~ms}$ and $\Delta f=400 \mathrm{MHz}$. The targets are resolved if $\Delta f$ is increased.

The expression (12) of the range resolution is a theoretical relationship; it assumes a perfect linear modulation of the transmitted signal.

\subsection{Simultaneous Range and Velocity Processing}

Signal model (3) assumes that the configuration is static: stationary radar and no moving targets. If this assumption is not true, it is necessary to take into account the radial velocities introduced by the relative movements between radar and the environment. In that case, the beat signal $s_{b}$ obtained with a sawtooth modulation can be expressed as [20],[24]

$$
s_{b}(t)=k \sum_{i} a_{t} a_{r i} \cos (2 \pi \underbrace{\left(2 \Delta f f_{m} \frac{r_{i}}{c}+2 f_{0} \frac{V_{r i}}{c}\right)}_{f_{b i}} t+\Phi_{i}),
$$

where $v_{r i}$ is the radial velocity between radar and target $i$. The beat frequency $f_{b i}$ is the sum of two components

$$
f_{b i}=\underbrace{\left(2 \Delta f f_{m} \frac{r_{i}}{c}\right)}_{f_{r}}+\underbrace{\left(2 f_{0} \frac{V_{r i}}{c}\right)}_{f_{d}} .
$$

The first part $f_{r}$ of (14) only depends on the range $r_{i}$, and the second part $f_{d}$ is the Doppler shift 
induced by the radial velocity $V_{r i}$. If $V_{r i}=0$, one can see that $f_{b i}$ is proportional to the radar-target distance $r_{i}$. Figure 5 is a geometrical illustration of the Doppler shift on the time-frequency evolution of the received signal: the received signal highlights a time delay $\tau_{i}$ corresponding to the radar-target distance $r_{i}$, and a vertical shift due to the frequency Doppler $f_{d}$ introduced by $v_{r i}$.

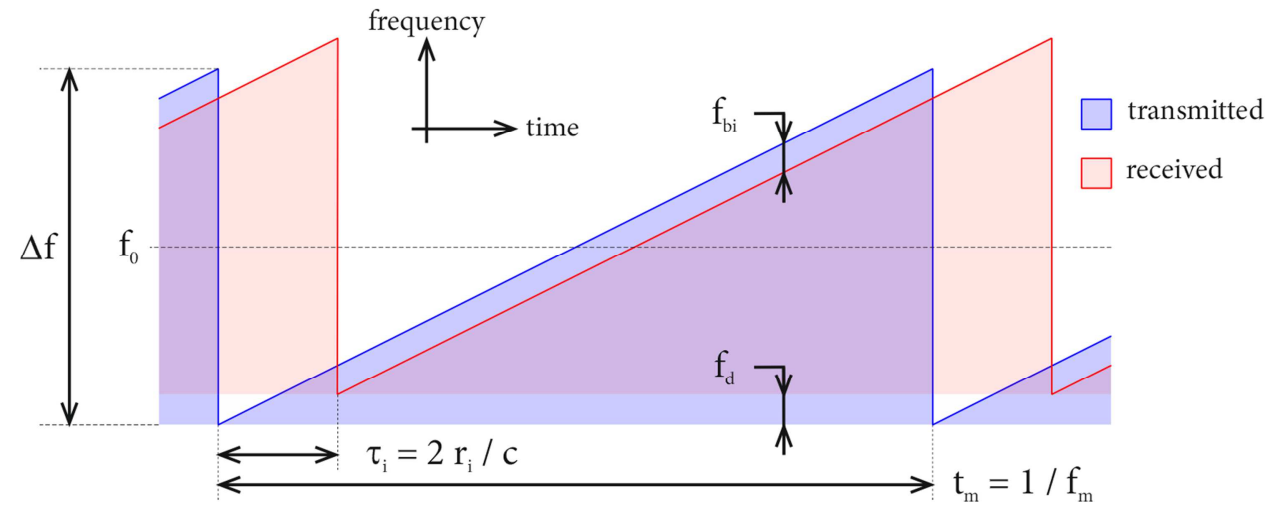

FIGURE 5: Sawtooth modulation in presence of radial velocity. When considering a target located at range $r_{i}$ with a radial velocity $V_{r i}$, the received signal highlights a time delay $\tau_{i}$ corresponding to the radar-target distance $r_{i}$, and a vertical shift due to the frequency Doppler $f_{d}$ introduced by $v_{r i}$.

The expression of the beat frequency $f_{b i}$ in (14) indicates that $f_{b i}$ depends simultaneously on distance $r_{i}$ and radial velocity $V_{r i}$. We obtain one equation with two unknowns $\left(r_{i}\right.$ and $\left.V_{r i}\right)$ : a sawtooth modulation highlights a range-velocity ambiguity. In that case, without a priori knowledge on $r_{i}$ or $V_{r i}$, the measurement of $f_{b}$ does not allow an unambiguous calculation of $r_{i}$ and $v_{r i}$ :

The simpler way to avoid this range-velocity ambiguity is to generate a triangular modulation law, including positive (upslope) and negative (downslope) slopes. Such a modulation law is presented in Figure 6. One can see in Figure 6 that the Doppler frequency $f_{d}$ is added or subtracted to the beat frequency part introduced by the radar-target distance, depending of the sign of the slope. This behavior leads to separate the calculation of the beat frequency over the positive and negative parts of the modulation.

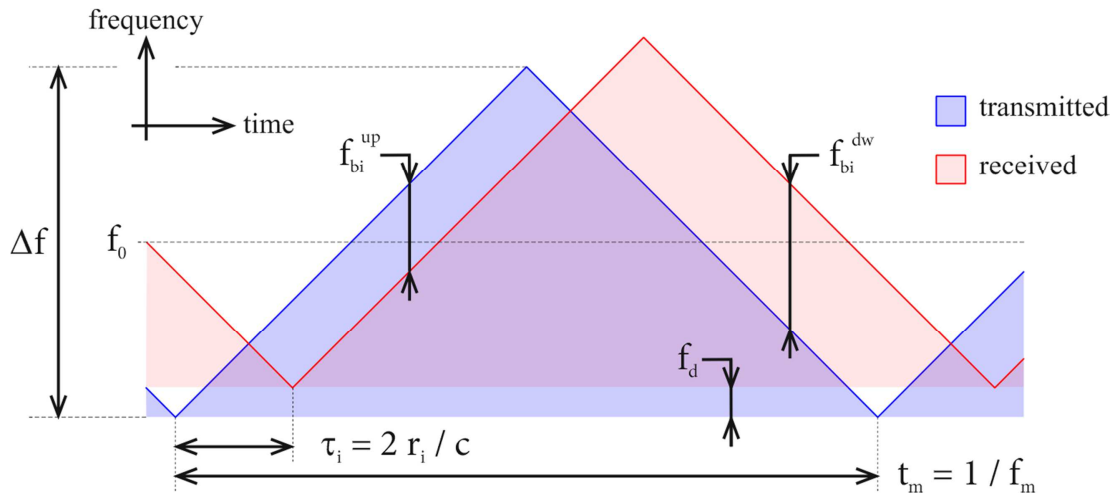

Figure 6: Triangular modulation law. The Doppler frequency $f_{d}$ is added or subtracted to the beat frequency introduced by the radar-target distance, depending of the sign of the modulation slope.

If $i$ target are located at ranges $r_{i}$ from the radar, with radial velocities $V_{r i}$, the beat signal is given by $[20],[24]$

$$
s_{b}(t)=k \sum_{i} a_{t} a_{r i} \cos (2 \pi \underbrace{\left(4 \Delta f f_{m} \frac{r_{i}}{c} \pm 2 f_{0} \frac{V_{r i}}{c}\right)}_{f_{b i}} t+\Phi_{i}),
$$


and the upslope and downslope beat frequencies can be written as

$$
\begin{aligned}
& f_{b i}^{a p}=4 \Delta f f_{m} \frac{r_{i}}{c}+2 f_{0} \frac{V_{r i}}{c}, \\
& f_{b i}^{d w}=4 \Delta f f_{m} \frac{r_{i}}{c}-2 f_{0} \frac{V_{r i}}{c} .
\end{aligned}
$$

The sum of (16) and (17) allows computing the range $r_{i}$ of the target, while the difference provides a calculation of the radial velocity $V_{r i}$

$$
\begin{gathered}
r_{i}=\frac{c}{8 \Delta f f_{m}}\left(f_{b i}^{u p}+f_{b i}^{d W}\right), \\
V_{r i}=\frac{c}{4 f_{0}}\left(f_{b i}^{u p}-f_{b i}^{d W}\right) .
\end{gathered}
$$

The sign of the radial velocity $V_{r i}$ depends on the moving direction of the target versus radar: negative sign if the target is approaching; positive sign if the target is moving away.

\subsubsection{Velocity Resolution}

The Doppler frequency $f_{d}$ is added or subtracted to the beat frequency part introduced by the radartarget distance, depending of the sign of the slope. Thus, the frequency shift $d f$ between the upslope and downslope spectra can be expressed as

$$
d f=f_{b}^{u p}-f_{b}^{d w}=2 f_{d}=4 f_{0} \frac{V}{c} .
$$

The velocity resolution $\delta V$ corresponds to the minimum measurable value of $d f$. With a triangular modulation, the signal is observed twice during the modulation period $t_{m}$, upslope and downslope, thus

$$
\min (d f)=\frac{2}{t_{m}}=2 f_{m}
$$

Finally, substituting (21) in (20), the expression of the velocity resolution $\delta_{V}$ is obtained

$$
\delta_{V}=\frac{c f_{m}}{2 f_{0}} .
$$

From (22), it can be seen that $\delta_{V}$ is inversely proportional to the carrier frequency $f_{0}$ and to the observing time $t_{m}$.

\subsubsection{Limitations of the Triangular Modulation Law for Velocity Measurement}

If theoretically the triangular modulation law allows the measurement of the radial velocities of the targets, its implementation remains a complex operation in multi-target environments. This difficulty is well illustrated with the simulated radar spectra in Figure 7 (radar parameters used are listed in Table 1).

In Figure 7 (a), a moving target $A$ with radial velocity $V_{A}=+5 \mathrm{~m} / \mathrm{s}$ is located at range $r_{A}=50 \mathrm{~m}$ in front of the radar. The radial velocity $V_{A}$ introduces the difference between upslope and downslope spectra. Range and velocity of the target are computed from (18) and (19). In this first example, the 
peak matching is simple and leads to a good estimation of the range and velocity of the target (see table in Figure 7 (a)).

In Figure 7 (b), two targets are located in front of the radar: target $A$ with radial velocity $V_{A}=+5 \mathrm{~m} / \mathrm{s}$ at range $r_{A}=50 \mathrm{~m}$; target $B$ with radial velocity $v_{B}=-5 \mathrm{~m} / \mathrm{s}$ at range $r_{B}=47 \mathrm{~m}$. In this example, there are two possibilities of peak matching and no way to find the good one without a priori knowledge on the targets behavior. The table in Figure 7 (b) shows the computed range and velocity for both associations: the wrong association (gray shaded area of the Table) leads to the detection of the targets with incorrect ranges and velocities.

The use of a triangular modulation law is further complicated in real environment by the presence of a large number of targets which increase the number of combinations between peaks, and hence the possible number of false associations. Some solutions have been developed to solve this problem. In [26], a fuzzy logic controller is used to match the upslope and downslope peaks, using similarity rules between peaks. However, considering the movement of the robot and the delay $\left(t_{m} / 2\right)$ between the upslope and downslope parts of the modulation, the profile of the upslope and downslope spectra can be very different. These differences, introduced by the coherent sum of the multiple elementary reflectors present at the same time in the antenna beam (Speckle effect), make problematic a correct peak matching.

It is necessary to use more complex modulation laws to obtain unambiguous range and velocity measurements simultaneously. Such a modulation law is described in [27] within the framework of automotive applications. This modulation combines FSK (Frequency Shift Keying) and LFM (Linear Frequency Modulation) waveforms: the waveform consists of two LFM up-chirp signals transmitted in an intertwined sequence, where the first stepwise frequency modulated sequence is used as a reference signal while the second up-chirp signal is shifted in frequency. We have developed a similar approach, with the combination of FH (Frequency Hopped) and LFM waveforms resulting in two intertwined linear waveforms [24].

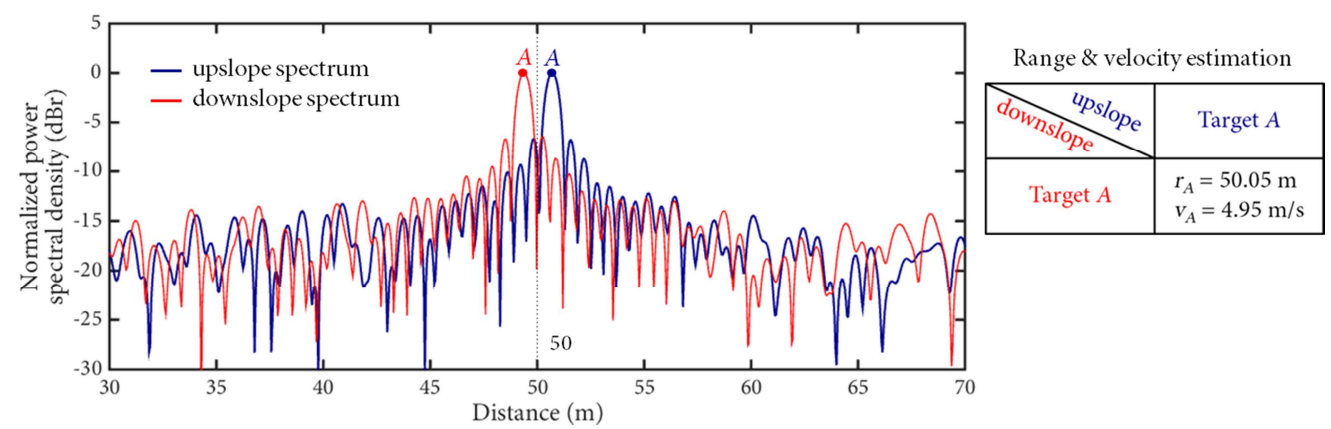

(a)

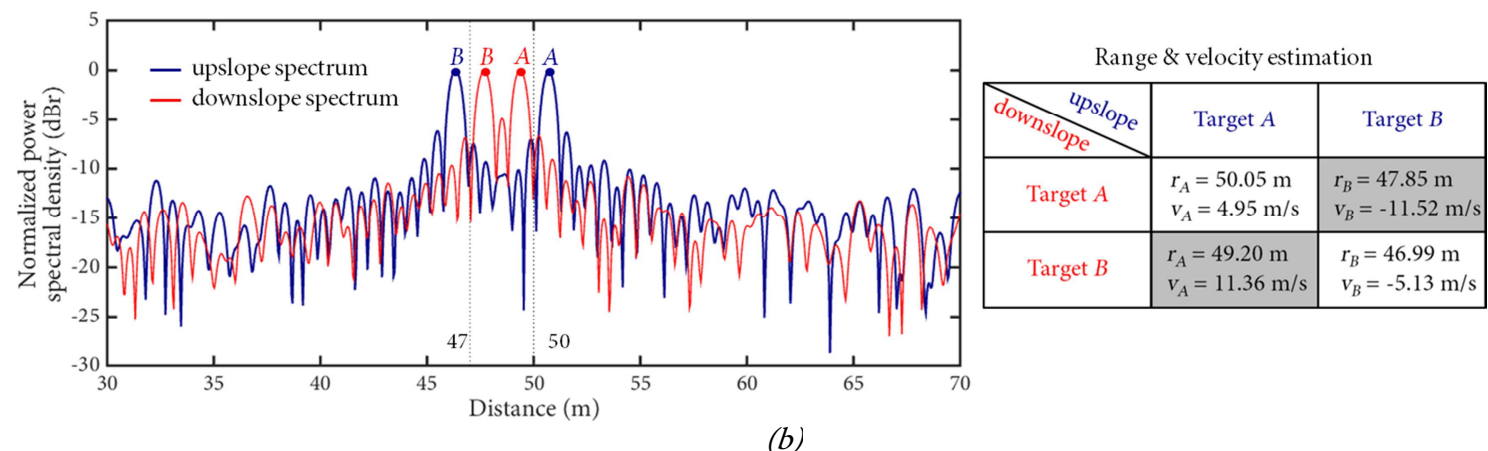

FIGURE 7: Simulation of radar spectra obtained with a triangular modulation law. Upslope and downslope spectra are computed in order to estimate simultaneously the range and velocity of the targets. (a) One target is located at range $50 \mathrm{~m}$, with a radial velocity of $+5 \mathrm{~m} / \mathrm{s}$. (b) Two targets A and B are located at range $50 \mathrm{~m}$ and $47 \mathrm{~m}$, with radial velocities of $+5 \mathrm{~m} / \mathrm{s}$ and $5 \mathrm{~m} / \mathrm{s}$ respectively. The wrong peaks association (gray shaded area of the Table) leads to an incorrect estimation of ranges and velocities. 
In summary, the Doppler shift introduced by the radial velocity between radar and targets can be taken into account with several approaches when considering FMCW radars:

- with a sawtooth modulation such as that used by PELICAN radar, the Doppler shift cannot be directly estimated. In that case, it is necessary to use a proprioceptive sensor to measure the velocity of the robot and at the same time assume a static environment (i.e. no moving elements in the environment). With the measurement of the velocity, one can estimate the Doppler shift $f_{d}$, and the beat frequency $f_{b}$ can then be corrected in order to compute the radar-target distance. But this solution remains limited because it is inefficient to correct the Doppler shift introduced by moving targets.

- the triangular modulation described in section 2.3 can be used to measure directly the Doppler shift. Radar spectra are computed for the upslope and downslope parts of the modulation, and a peak matching allows recovering distances and radial velocities of the targets. However, this approach suffers from major drawbacks: (i) in multi-target environments (classical situation for a robot which moves in an outdoor environment), the targets configuration can lead to ambiguous situations, where peaks association strategies fail; (ii) and due to fading effects, upslope and downslope spectra can be slightly different, making the peak matching problematic or impossible.

- more complex modulation laws can be used to obtain unambiguous range and velocity measurements simultaneously in all situations. We have developed the intertwined linear frequency modulation, which combines frequency hopped and linear frequency modulation waveforms. This solution has been modeled and simulated, and it is presented in more detail in [24]. Potentially very efficient, that solution implies the transmission of synchronized intertwined signals, which will lead to much more complex and expensive microwave architectures than those developed for sawtooth modulation.

Considering that we are not involved in mobile object tracking applications (MOT), the use of a sawtooth modulation is possible, and we will use this approach in the remainder of this paper: classical sawtooth modulation + static environment assumption + proprioceptive sensor to measure the velocity of the robot. One can also notice that the relative simplicity of implementation of sawtooth modulation will allow developing a compact and low-cost microwave architecture.

\section{Choice of the Carrier Frequency}

The choice of the carrier frequency $f_{0}$ of a radar system is not a trivial process, considering its influence on numerous parameters such as maximum range, attenuation through the atmosphere, etc. Finally, the choice of $f_{0}$ is the result of a compromise between several criteria. These criteria include intrinsic parameters related to technological aspects, and extrinsic parameters related to specific constraints of the expected application.

\subsection{Detection of Small Objects - Penetration Capabilities - Roughness Effect}

The carrier frequency $f_{0}$ has a direct influence on target's detection and penetration capabilities. It is accepted that objects whose typical sizes are much smaller than the wavelength will not be detected. For example in radar remote sensing, a forest will be partially invisible at lowest frequencies such as $\mathrm{P}$ band (250-500 MHz), and only the trunks will be able to reflect the radar signal; but at X band (8$12 \mathrm{GHz}$ ) only the canopy will be detected because the size of leaves and branches are on the order of the wavelength [28].

The penetrating capabilities of radar signal are widely used by ground penetrating radars (GPR) [29]. The penetration of materials, soil, walls, etc. can be obtained through the use of frequency typically lower than $1 \mathrm{GHz}$. The penetration depth is between few decimeters for the highest frequencies $(0.9-2.6 \mathrm{GHz})$ and few meters for lower ones $(0.1-0.6 \mathrm{GHz})$. But due to their conductivity and dielectric constant, some materials such as wet soils or clay soils have strong attenuation capabilities that limit or completely prevent radar penetration. 
Surface roughness is a dominant factor in determining the amplitude of the backscattered signal from a surface [30]. It measures the irregularity of surface compared to the wavelength (and the angle of incidence). This last point is fundamental: a surface can exhibit a "smooth" behavior at a radar wavelength, and "rough" behavior at a shorter wavelength. If a surface is smooth, the backscattered signal will only exhibit a reflection in the specular direction because the surface acts like a mirror for the incident radar signal. For a slightly rough surface, the level of energy reflected in the specular direction decreases while a diffuse reflection increases. And for very rough surface, a diffuse reflection only subsists.

\subsection{Radar Signal Attenuation through the Atmosphere}

Three elements are taken into account when considering radar signal attenuation in the air: (i) freespace path loss, (ii) absorption due to the presence of oxygen and water vapor molecules, and (iii) attenuation due to rain drops and dust or sand particles.

The equation of the free-space path loss (FSPL) [31] is given by

$$
F S P L=\left(\frac{4 \pi r f_{0}}{c}\right)^{2},
$$

where $r$ is the transmitter-receiver distance. The FSPL is proportional to the square of the distance $r$, and also to the square of the frequency $f_{0}$. The radar equation (7) can also be used to explain the influence of $f_{0}$ on the free-space path loss: if $f_{0}$ increases (i.e. $\lambda$ decreases), the transmitted power $p_{t}$ must be increased in order to maintain the same received power $p_{r}$.

Losses occur when the radar signal is absorbed by molecules of oxygen and water vapor. One can see in Figure 8 that the losses are greater at specific frequencies, corresponding to the mechanical resonant frequencies of the molecules [32].

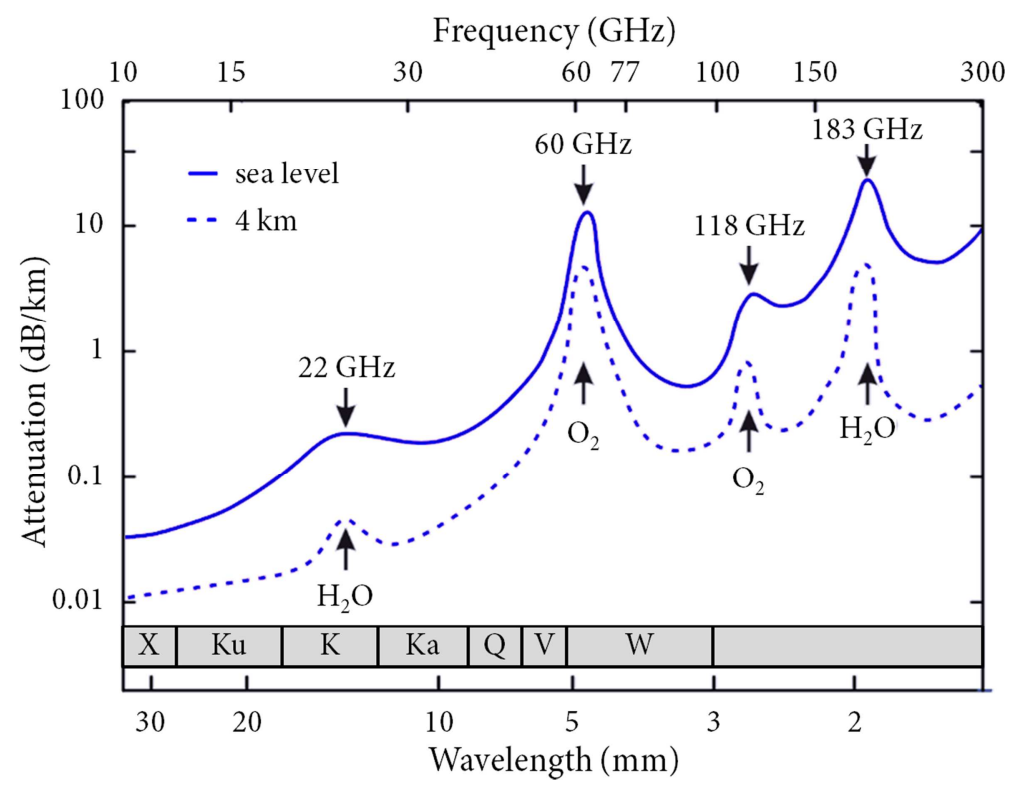

FIGURE 8: Specific attenuation due to the presence of oxygen and water vapor in the atmosphere.

The presence of rain drops, dust or sand particles also influences the radar signal attenuation. This problem has been widely studied for wireless communication applications [33]. Researches have shown that the shorter the wavelength, the more attenuation will be induced by absorption and scattering due to rain drops, dust and sand particles. But considering that radar-target distances involved in AGV applications are short (no more than a few hundred meters), attenuations caused by dust, rain or sand particles could be neglected in this particular case. 


\subsection{Radar Dimensions}

The general law concerning the radar dimensions can be summarized as follows: the higher the frequency, the lower the size of the radar. This behavior is particularly relevant for the dimensions of the antenna. A rough estimation of the beam width $\theta$ expressed in radians is given by the ratio of the wavelength $\lambda$ to the antenna size $d$ [22]

$$
\theta \approx \frac{\lambda}{d}
$$

From (24), it can be seen that the increase of $f_{0}$ (the decrease of $\lambda$ ) allows reducing $\theta$ for a given antenna size $d$. Or if $\theta$ is maintained constant, the increase of $f_{0}$ leads to build an antenna with smaller dimension.

\subsection{Doppler Shift}

From (4), one can notice that the Doppler shift has a direct influence on the beat frequency because the higher $f_{0}$, the higher the Doppler frequency induced by the radial velocity. In that sense, the choice of an adapted carrier frequency $f_{0}$ can help to increase or to reduce the effect of Doppler shift.

\subsection{Cost and Availability of Microwave Components}

Cost and availability of microwave components (oscillator, antenna, etc.) are still constraints for the development of new devices and applications in the industrial or research domains. It is a fluctuating situation, because due to the emergence of new markets, up-to-date components are regularly proposed by dealers. For example with the development of automatic cruise control or anti-collision applications in the automotive sector, high frequency components (above $70 \mathrm{GHz}$ ) are now available for "low-cost" civilian applications. Another constraint that must be taken into account during the research and development stages is the cost of the laboratory instruments, that increases in direct relation with the frequency $f_{0}$.

\subsection{Allocation of Frequency Spectrum}

Devices and systems require authorizations to radiate RF energy. These authorizations are managed by several organizations, at national and international levels. For example, the International Telecommunication Union (ITU) [34] is in charge of the allocation of global resources such as radiofrequency spectrum. And in France, the use of the radio-frequency spectrum is controlled by the French Telecommunications Regulatory Authority (ARCEP) [35]. All these regulation laws lead to the definitions of limitations such as prohibited frequency bands, or bandwidth and power limitations.

\section{Construction of $2 \mathrm{D}$ images}

Our objective is to develop radar and use it to build a representation (an image) in the vicinity of the robot. If several approaches can be used to build radar images, it is possible to define two categories depending on the use of Real Aperture or Synthetic Aperture Radars.

Synthetic Aperture Radar (SAR) is widely used in the domain of aerial or satellite radar remote sensing, for the observation of Earth or other planets of the solar system [36],[37]. Because of the radar-target distances considered in airborne or spaceborne applications, the antenna aperture leads to a wide radar footprint and a poor angular resolution. As the antenna size cannot be increased at will in order to reduce the antenna aperture, SAR systems utilize the flight path of the platform (aircraft or satellite) to emulate (to synthesize) an extremely large antenna. SAR works like a phased-array antenna, but contrary of a large number of parallel antenna elements of a phased array, SAR uses one antenna in a time-multiplex process: the different geometric positions of the antenna elements are the result of the displacement of the platform. For example, the ERS-2 satellite (launched in 1995; end of 
mission in 2011) was equipped with a $10 \mathrm{~m} \mathrm{SAR}$ antenna in C-band (5.3 GHz), which corresponds to a beamwidth of $0.33^{\circ}$. Considering an orbital altitude of $785 \mathrm{~km}$ and an incidence angle of $23^{\circ}$, the ERS-2 footprint highlights a size of about $4900 \mathrm{~m}$ in the azimuthal dimension: the SAR processing allows obtaining an azimuth resolution between 10 and $30 \mathrm{~m}$. The SAR signal processing uses magnitude and phase of the received signals over successive radar acquisitions. It implies an exact knowledge of the flight path and velocity of the platform. Considering a robot following an unknown trajectory in an unknown environment (non-flat natural environment), an effective and precise measurement of the robot trajectory is by itself a non-trivial problem. Moreover, SAR processing requires a large amount of data storage capacity and processing power, which make difficult the development of real-time applications. For all these reasons, we selected a Real Aperture Radar solution for the radar image construction.

In a Real Aperture Radar (RAR), the angular resolution is directly related to the real aperture of the antenna (in contrast to SAR systems) [38]. It means that the angular resolution is very coarse for longrange applications. But this characteristic may not be a limitation for short range applications we are interested in. RAR systems are still widely used on surveillance and air traffic control applications or marine radar systems onboard ships for collision avoidance and navigation at sea.

The adopted solution is inspired of marine radar systems. This solution uses a fan-beam antenna, which produces a main beam with a narrow beamwith in the horizontal plane (the azimuth) and a wider beamwidth in the vertical plane (the elevation). A simulation of the antenna radiation pattern is presented in Figure 9. This antenna has a half power $(-3 \mathrm{~dB})$ aperture of $5^{\circ}$ and $25^{\circ}$ (azimuth and elevation planes respectively).

The radar is positioned on the top of a platform (robot, vehicle, etc.) in order to have an unobstructed view. A typical positioning of the radar is presented in Figure 10. The antenna rotates in the horizontal plane over the full $360^{\circ}$ in order to build a panoramic image of the environment. The antenna is equipped with an encoder in order to measure the pointing direction $\theta$ in a robot-fixed reference frame. The use of a fan-beam antenna is a main characteristic and a major limitation of the developed solution. The use of a large elevation beamwidth is a purpose in order to make the radar robust to some severe positioning variations in pitch and roll of the robot when it navigates in natural and non-flat environments. But at the same time the elevations or heights of the targets cannot be determined: with such a fan-beam antenna, the radar can only build 2D images of the environment. The information will be displayed in PPI (Plan Position Indicator) format, which is a common type of radar display: the detected elements are localized through (azimuth $\theta$, distance $r$ ) polar coordinates in a $\left(x_{r}, y_{r}, Z_{r}\right)$ vehicle-fixed reference frame. 
Author-produced version of the article published in Robotics and Autonomous Systems, 2016, 81, 1-16.

The original publication is available at http://www.sciencedirect.com/ doi : 10.1016/j.robot.2016.04.001

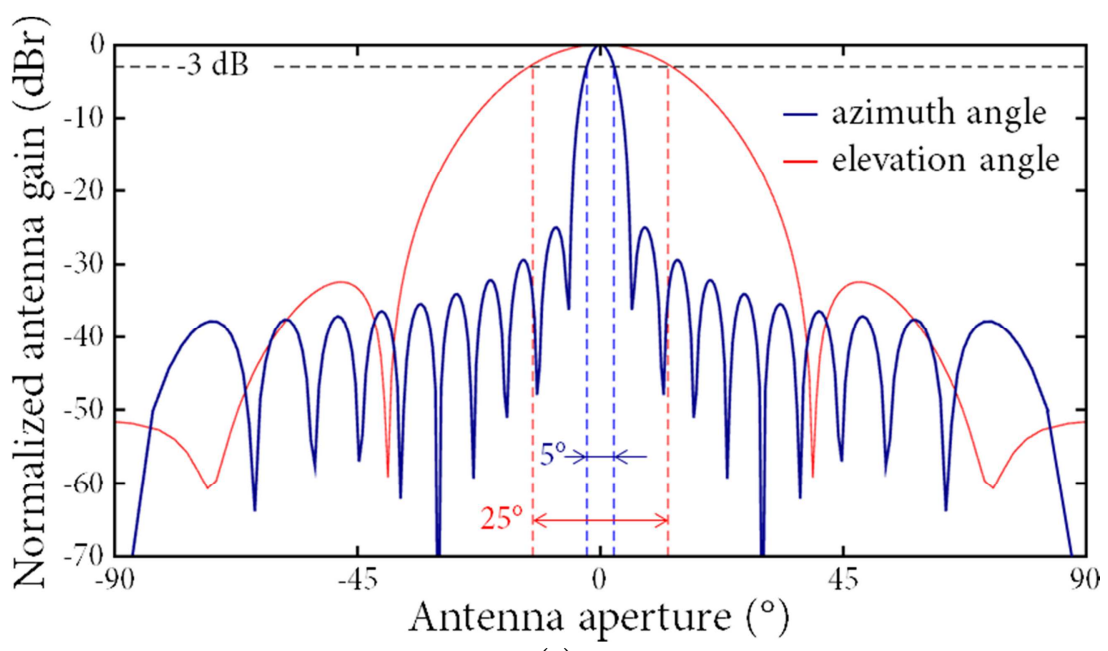

(a)

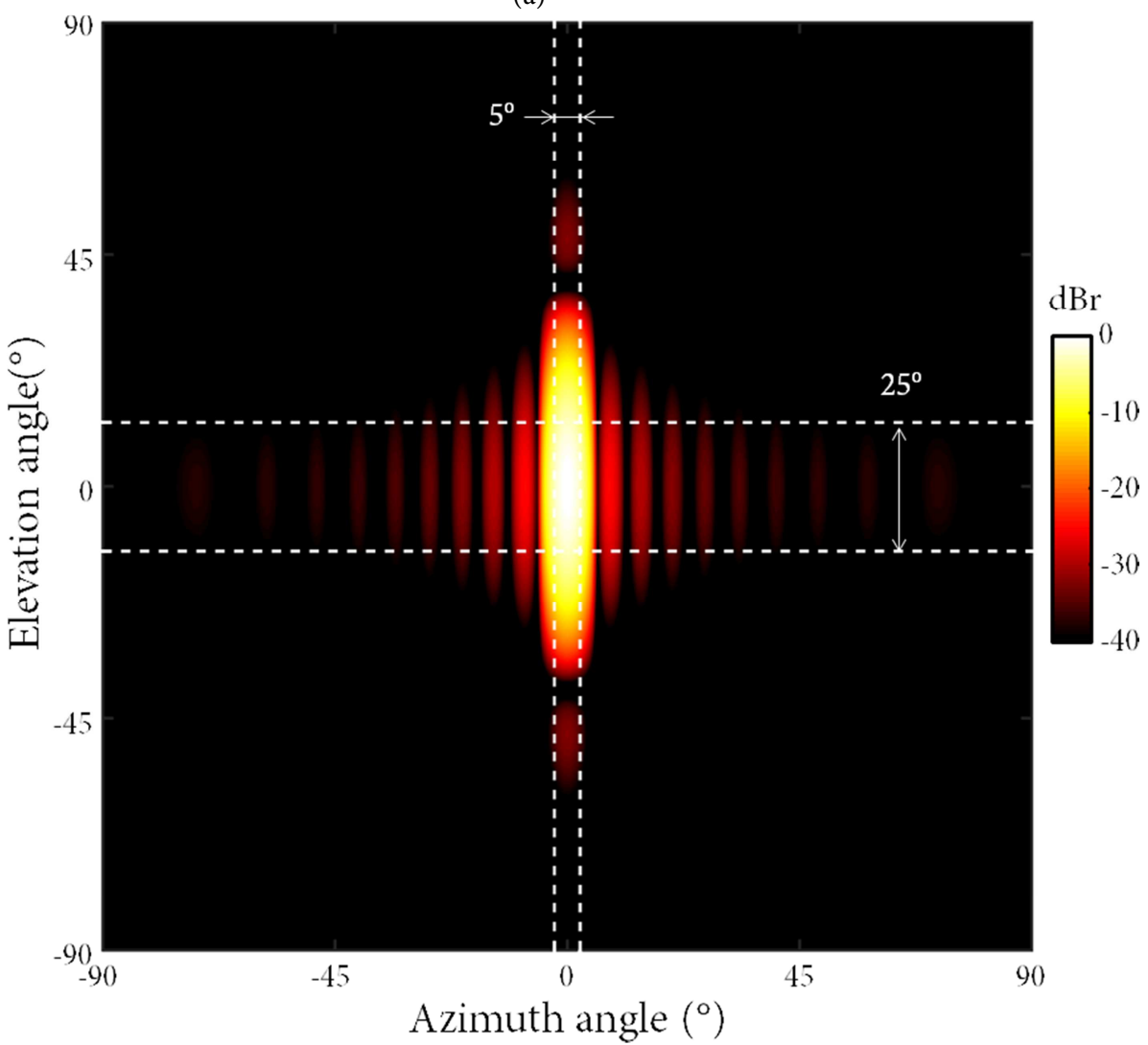

(b)

FIGURE 9: Simulated radiation pattern of a fan-beam antenna. Half-power beamwidth: $5^{\circ}$ in the azimuth plane, $25^{\circ}$ in the elevation plane. (a) Normalized gain of the antenna along the main azimuth and elevation axis. (b) 3D resulting antenna radiation pattern. 


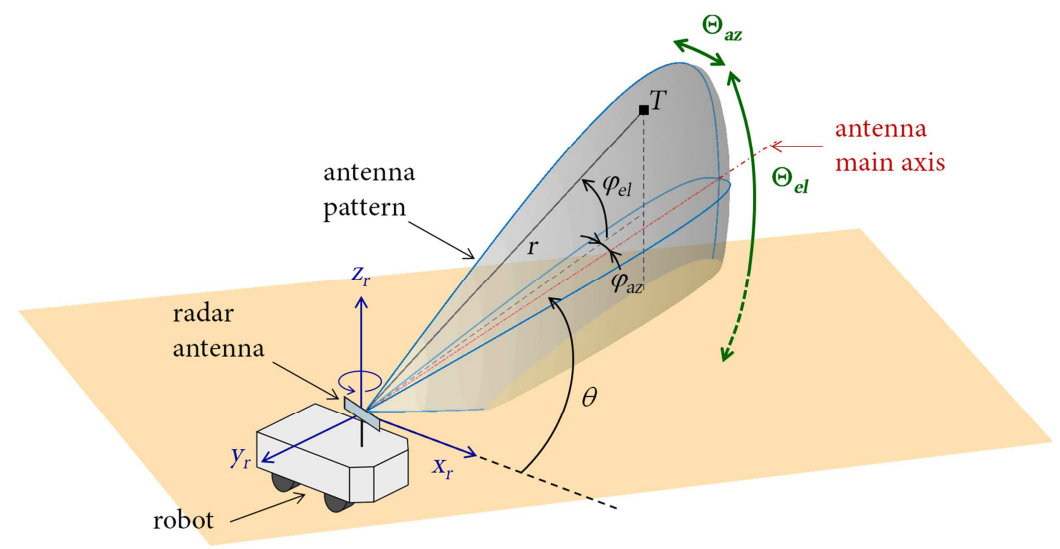

FIGURE 10: Position of radar on a robot. Radar is typically located at a height $h_{r}$ between $1 \mathrm{~m}$ and $3 \mathrm{~m}$ above the ground, depending on the dimensions of the robot. $\Theta_{a z}$ and $\Theta_{e l}$ are the half-power aperture angles of the antenna (azimuth and elevation respectively). A target $\mathrm{T}$ situated in the antenna beam is localized through its polar coordinates $(\theta, \mathrm{r})$. The elevation angle and azimuth angles $\left(\varphi_{e l}, \varphi_{a z}\right)$ of target $\mathrm{T}$ within the antenna beam are unknown parameters.

An example of simulated 2D radar image based on the rotation of a fan-beam antenna is presented in Figure 11 and Figure 12. The main characteristics of the simulated radar and antenna are shown in Table 1 and Table 2. The radar uses a linear sawtooth modulation described in Figure 2 and Figure 5.

TABLE 2: Characteristics of the simulated antenna.

\begin{tabular}{lc}
\hline Half-power aperture & \\
- azimuth plane $\Theta_{a z}$ & $5^{\circ}$ \\
- elevation plane $\Theta_{e l}$ & $25^{\circ}$ \\
Rotation velocity & $60 \mathrm{rpm}$ \\
Gain & 100 \\
\hline
\end{tabular}

Figure 11 shows the simulated environment. The environment is discretized with a Delaunay triangulation. Each elementary triangle is characterized by its $3 \mathrm{D}$ position, a normal vector $\vec{n}$ to the surface and a backscatter coefficient $\sigma_{0}$. The radar is in a fixed position, $3 \mathrm{~m}$ above the ground (blue square). The environment is modeled with flat and hilly terrains. Using cubic shapes, several buildings, walls and poles are positioned in the field of view of the radar. The antenna achieves a complete rotation in one second $(60 \mathrm{rpm})$. In this example, the maximum range $r_{\max }$ is $100 \mathrm{~m} . r_{\max }$ is set by the sampling frequency $f_{s}$ which determines the maximum measurable beat frequency $f_{b \max }$, and consequently the maximum measurable radar-target distance. The dashed circle in Figure 11 represents this maximum range.

A radar signal and a radar spectrum are computed at each degree of antenna rotation, taking into account pitch, roll and heading of the vehicle. The discrete points of the environment which are intercepted by the antenna pattern are considered as elementary scatterers and used to compute the corresponding radar signal based on (13). A range compensation is applied in order to eliminate the effect of the distance on the amplitude of the radar signal: the term $r^{-2}$ in (8) is compensated, and the amplitude received from a target becomes proportional to the square root of its radar cross section $\sigma$, independently of the distance $r$. A PPI radar image is obtained at the end of each antenna rotation, with the merge of 360 radar spectra. The radar image obtained is shown in Figure 12, in polar coordinates in Figure 12(a) or in Cartesian coordinates in Figure 12(b). In polar coordinates $(\theta, r)$, each column of data corresponds to a single radar spectrum (i.e. one antenna pointing direction). Polar coordinates $(\theta, r)$ can be transformed to Cartesian coordinates $(x, y)$ for visualization purposes or robot navigation. This transformation can be source of confusion due to the antenna beamwidth: for example, point targets have the same angular occupancy independent of the range in polar coordinates, but the corresponding echoes in Cartesian coordinates highlight different spatial 
occupancies. This phenomenon is well-illustrated in Figure 12 with echoes from the poles (see marks $P_{1}$ and $\left.P_{2}\right)$.

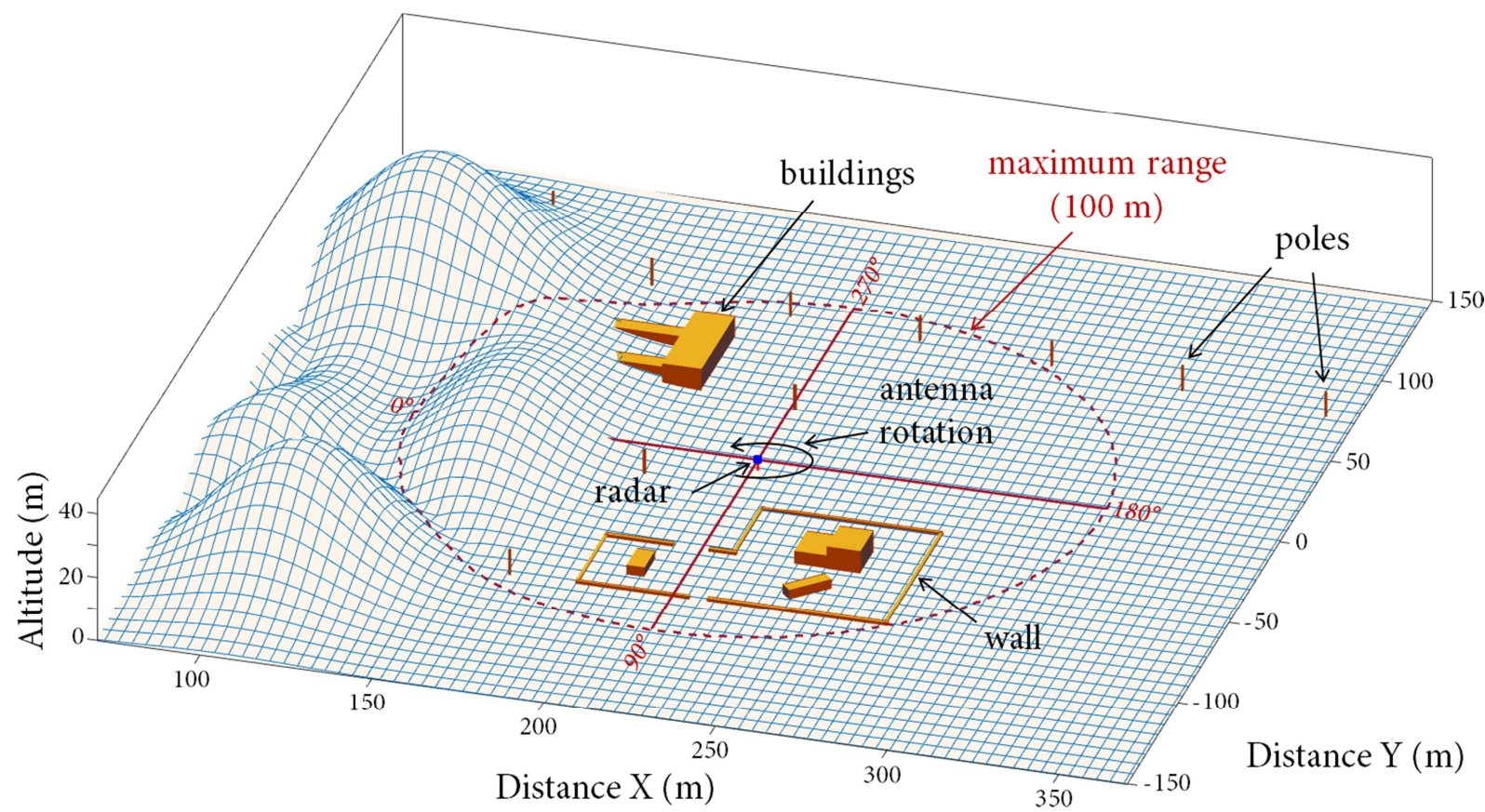

FIGURE 11: Example of environment modeling. The radar is in a fixed position, $3 \mathrm{~m}$ above the ground. The environment includes hilly and flat terrains. Cubic structures are used to simulate buildings, walls or poles. The maximum measurable distance is set to $100 \mathrm{~m}$ (represented by the dashed circle).

One can observe an empty area of radius $10 \mathrm{~m}$ approximately around the radar (mark $A$ ), due to the height of the radar and the elevation beamwidth of the antenna. Shadowing effects can be seen behind the buildings and walls (marks $B, C$ and $D$ for example) or behind the hills (mark $E$ ). The granular noise that can be observed on the ground is an illustration of the Speckle effect. The Speckle effect is an inherent process to any radar image construction, and it is introduced by phaseinterference effects (fading). Suppose that $N$ elementary scatterers are present within the radar footprint: the $N$ scattered signals are summed coherently to produce the measured radar signal. The scattered signals are summed constructively or destructively, depending on their relative phase, and the result is a random amplitude modulation of the radar signal, which introduces a grainy salt-andpepper pattern in the radar image. The same effect can be observed with the echoes from the buildings or the walls.

\subsection{Slant Range Error}

The constructed 2D radar image can be seen as the projection of all targets on a reference plane (the rotation plane of the antenna). Because the fan-beam antenna does not allow determining the altitude of a target, the target ground range (i.e. the range between the radar and the ground projection of the target, also called down range) is unknown. Consider an antenna with a rotation plane parallel to the ground, and a target $T$ with a slant range $r_{s}$ and an elevation angle $\varphi_{e l}$. In that case, the ground range $r_{g}$ can be written as

$$
r_{g}=r_{s} \cos \left(\varphi_{e l}\right) .
$$




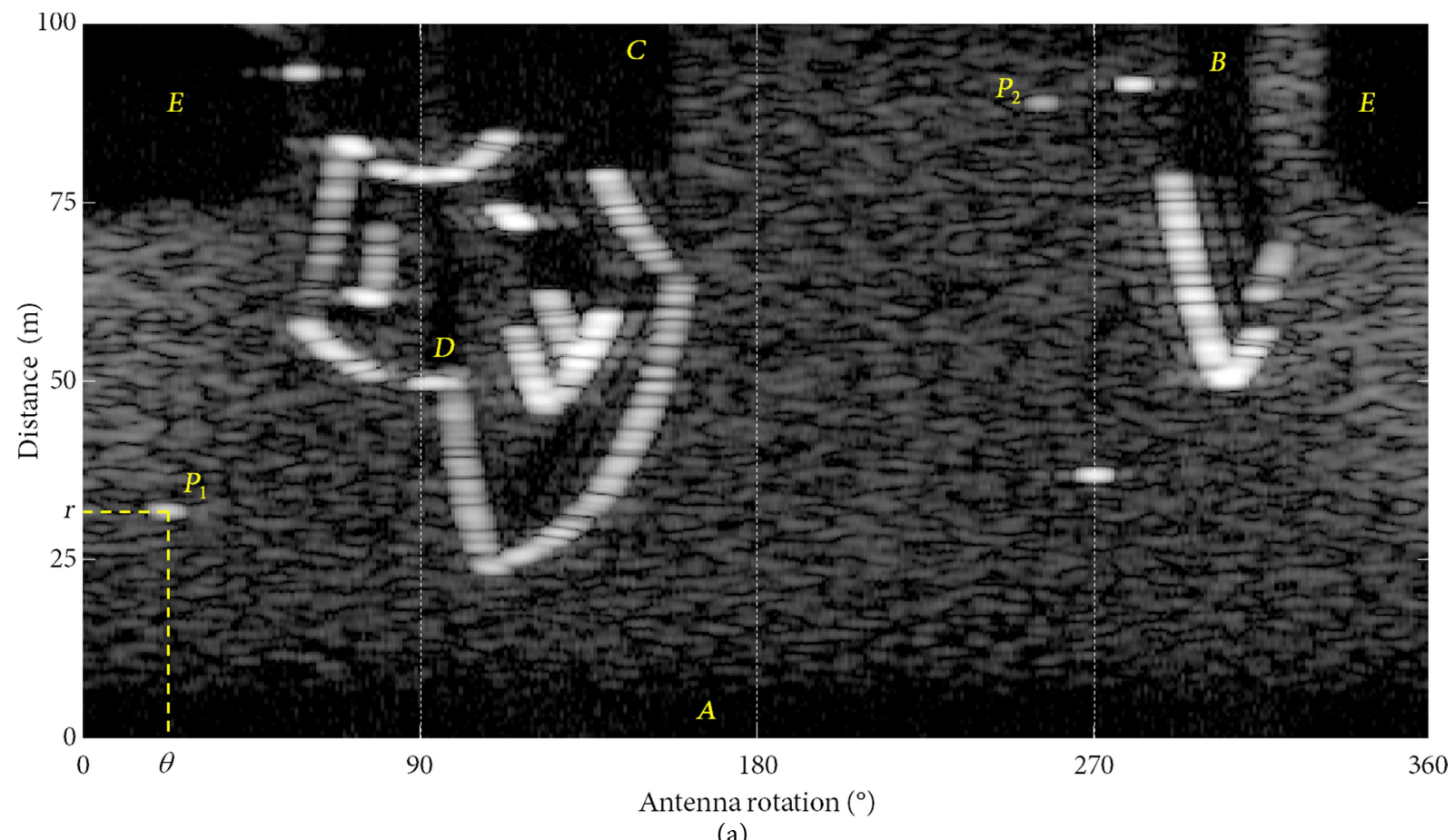

(a)

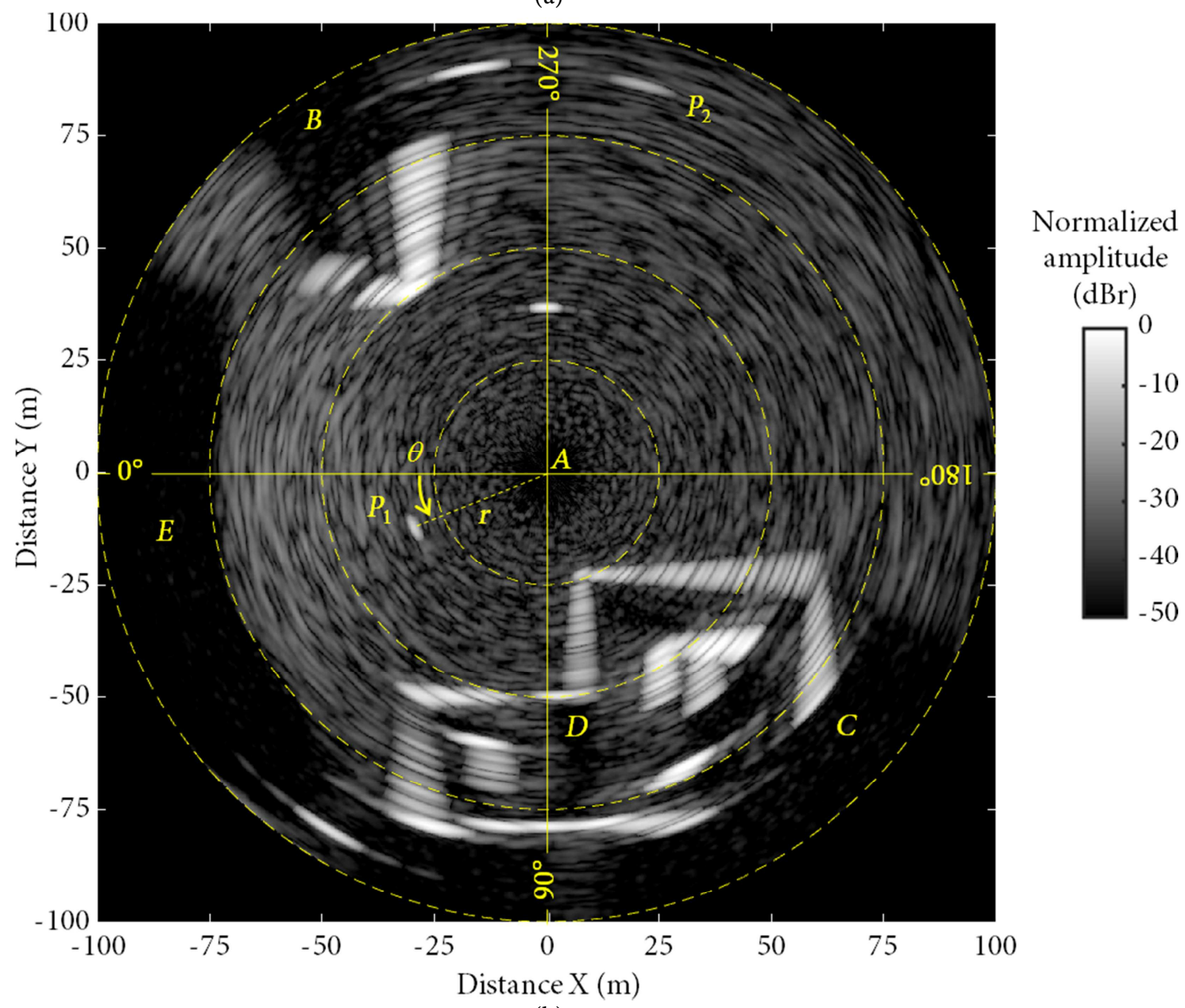

(b)

FIGURE 12: Simulated 2D radar image, in polar coordinates (a) and Cartesian coordinates (b). Mark A indicates the dead zone introduced by the radar height and the antenna elevation aperture. Shadowing effect can be observed behind the buildings (marks B and C), behind the walls (mark D) and behind the hills (mark E). $P_{1}$ and $P_{2}$ are echoes from poles (considered as point targets): they highlight the same angular occupancy in polar coordinates, but different spatial occupancies in Cartesian coordinates due to range difference. The grainy salt-and-pepper texture in the radar image is caused by the Speckle effect. 
(25) is a simple form equation because we assume that the measured distances are short and it is not necessary to take into account the curvature of the earth. But the usefulness of (25) is to show that a fan-beam antenna introduces a range migration when the $2 \mathrm{D}$ projection is achieved, with a systematic overestimation of the ground distance (excepted when radar and target are at the same altitude, i.e. $\left.\varphi_{e l}=0^{\circ}\right)$. This problem is illustrated in Figure 13.

(a)

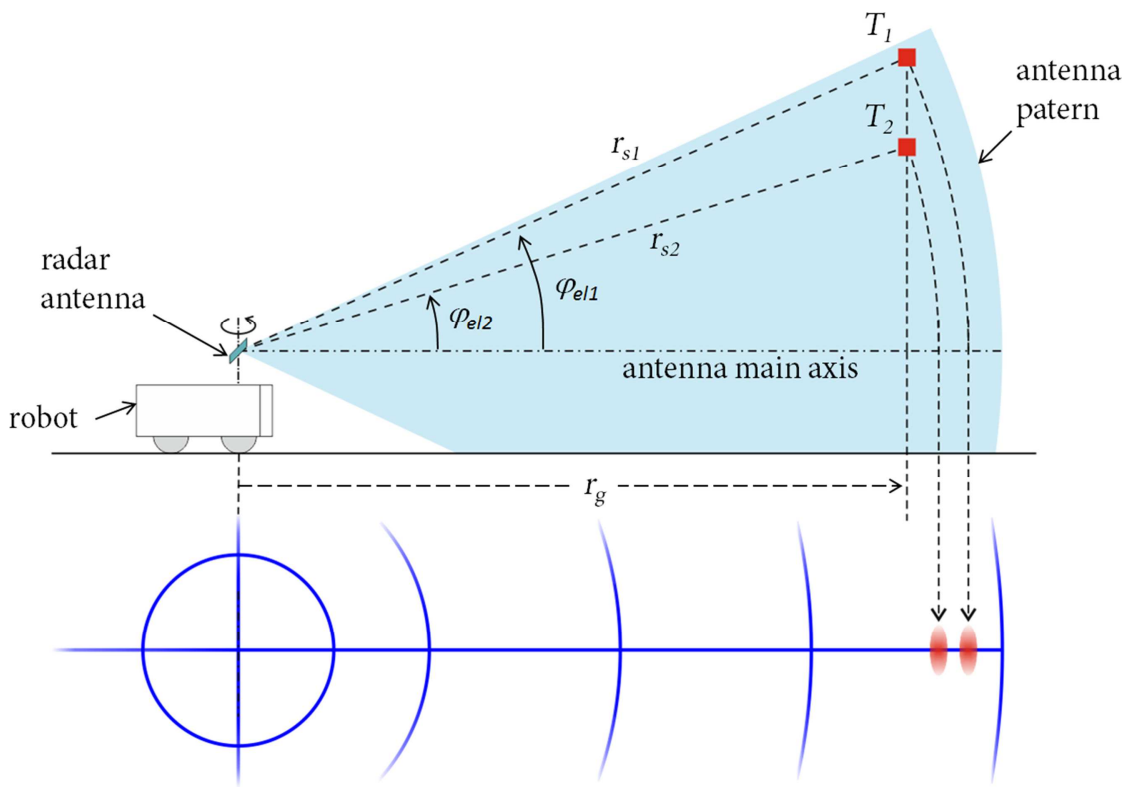

FIGURE 13: Illustration of the range migration introduced by an altitude difference. (a) Two targets $T_{1}$ and $T_{2}$ are at the same position in front of the radar (i.e. same ground distance $r_{g}$ ) with a difference in altitude (i.e. a difference in elevation angle $\varphi_{e l}$ ). $r_{s 1}$ and $r_{s 2}$ are the distances (slant ranges) between the radar and the targets. (b) The PPI representation highlights the difference between the measured slant ranges $\left(r_{s 1}\right.$ and $\left.r_{s 2}\right)$ and the ground range $r_{g}$.

A simulation of the range migration due to the altitude difference between radar and target, with radar characteristics described in Table 1 and Table 2, is shown in Figure 14. One can see that the range migration remains low for low elevation angles and low distances. The range migration increases as a function of the distance and altitude of the target: for a target with a slant range $r_{s}=90 \mathrm{~m}$ and an elevation angle $\varphi_{e l}=+10^{\circ}$ (i.e. difference in elevation of around $15.9 \mathrm{~m}$ between radar and target), the range migration is equal to $1.37 \mathrm{~m}$.

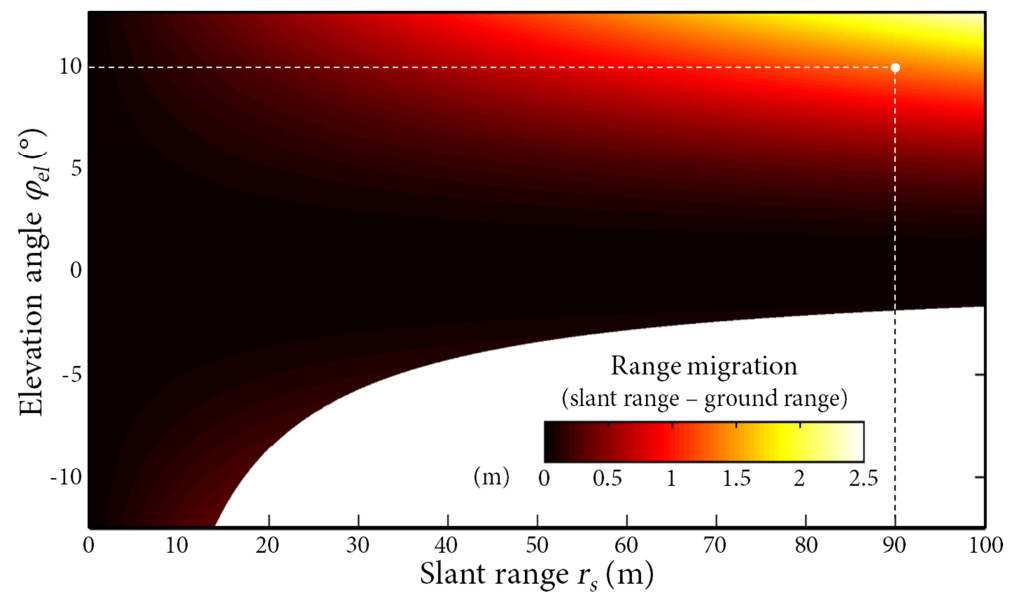

FIGURE 14: Simulation of the range migration as a function of slant range $r_{s}$ and elevation angle $\varphi_{e l}$. The range migration is the difference between the slant range $r_{s}$ and the ground range $r_{g}$. It is introduced by the altitude difference between the radar and the target. For this simulation, the aperture of the antenna in the elevation plane is $25^{\circ}\left( \pm 12.5^{\circ}\right)$ and the radar is positioned $3 \mathrm{~m}$ above the ground. 
In Air Traffic Control applications, the range migration problem is managed by the use of multiple synchronous 2D radars [39], or by the use of one 2D radar and some assumptions concerning the target attitude ("stable" trajectory, with constant velocity and altitude) [40]. As we have one radar, the only way to correct this range migration is to add knowledge about the environment during the $2 \mathrm{D}$ image construction. We have studied such an approach in [41]: the topography effects induced during the construction of a radar map are corrected with the use of a digital elevation model (DEM) of the environment.

\subsection{Platform motion correction}

The combined effect of antenna rotation and robot motion (translations + rotation) introduces distortions between the 360 successive radar spectra which are used to produce one radar image. This distortion effect is illustrated in Figure 15. One can see in Figure 15(b) that the detected points are improperly positioned if the movement of the robot is neglected. The correction is achieved by the use of two proprioceptive sensors which allow to estimate the trajectory of the robot during one antenna revolution: an odometer measures the longitudinal displacement, and a gyrometer measures the rotation. By combining data from the odometer and from the gyrometer with data from the antenna encoder, the 360 radar spectra can be projected in a common reference frame in order to build one revolution radar image.

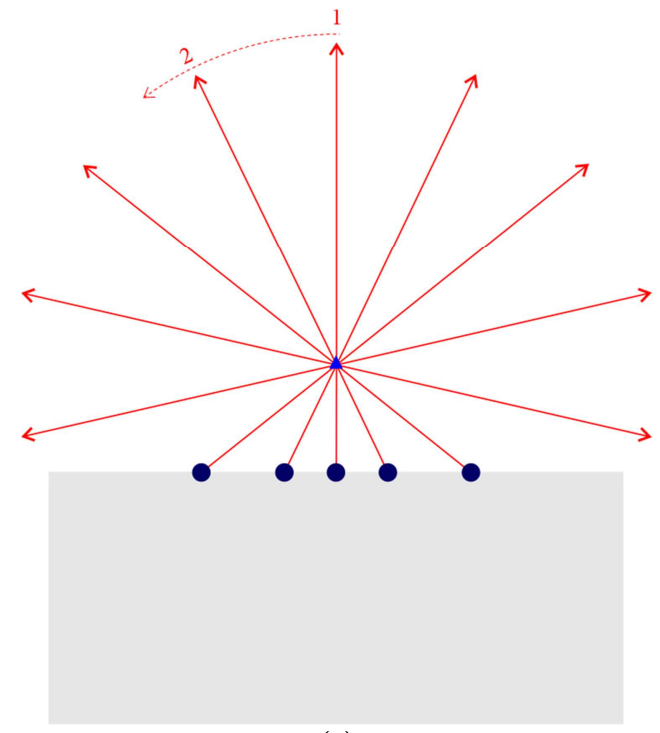

(a)

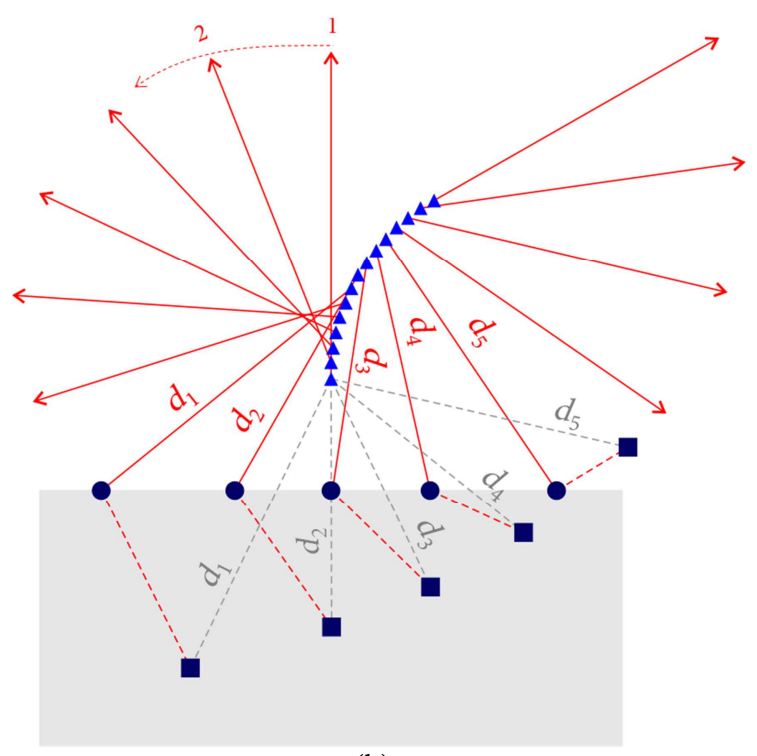

(b)

FIGURE 15: Illustration of the distortion introduced by the robot motion. The triangles indicate de successive positions of the radar, the arrows the successive antenna pointing directions. The circles localize the detected points in presence of a target. (a) Without robot motion. (b) With robot motion. If the robot motion is neglected, the detected points are improperly positioned (squares).

\subsection{Doppler correction}

From (14), one can see that the beat frequency $f_{b}$ is the sum of two components: the first one depends on the radar-target distance $r$ and the second is the Doppler shift $f_{d}$ introduced by the relative velocity $V_{r}$ between radar and the target. When considering a static environment (without moving targets), $V_{r}$ only depends on the robot velocity $v$ and on the angle $\theta$ between the direction of the robot and the antenna direction of propagation $(\theta$ is defined in a robot-fixed reference frame and is measured by the antenna encoder). Finally $f_{d}$ can be expressed as 


$$
f_{d}=\frac{2 f_{0}}{c} \underbrace{(v \cos (\theta))}_{V_{r}} \text {. }
$$

An illustration is given in Figure 16(a). The amplitude of the radial velocity $v_{r}$ is maximal for targets in the line of the robot trajectory (see $T_{1}$ and $T_{2}$ marks) and null for targets perpendicular to the robot trajectory (see $T_{3}$ and $T_{4}$ marks). When the robot is moving forwards, the measured radar-target distance is greater (respectively smaller) than the true distance for targets positioned in front of the robot (respectively behind the robot). In Figure 16(b), one can see that a robot velocity of $5 \mathrm{~m} / \mathrm{s}$ introduces a maximum shift distance of about $1.33 \mathrm{~m}$ with radar parameters listed in Table 1.

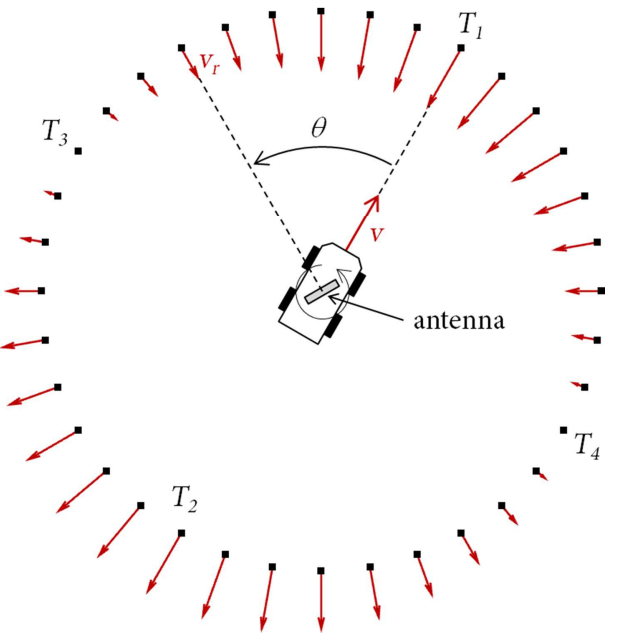

(a)

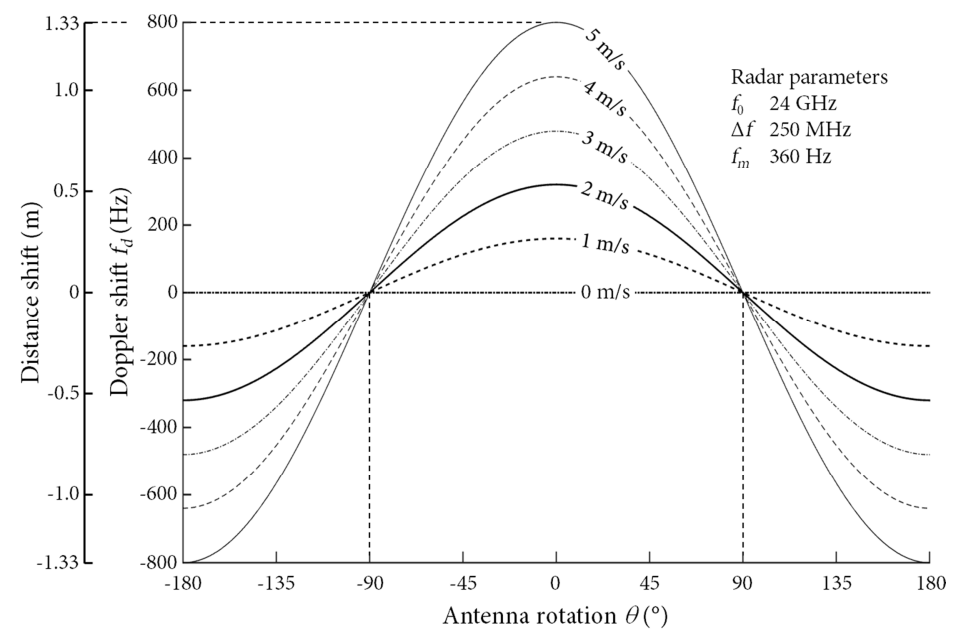

(b)

FIGURE 16: Radial velocity introduced by the robot motion. (a) Variation of the radial velocity $v_{r}$ as a function of the antenna rotation $\theta$. (b) Simulation of Doppler and distance shifts evaluated at several robot velocities. Radar parameters are listed in Table 1.

The radar spectra are shifted up or down depending on the sign and the amplitude of $v_{r}$. With a sawtooth modulation, $V_{r}$ cannot be isolated, and therefore it is necessary to measure the robot velocity $V$ with a proprioceptive sensor (for example with the odometer used to correct the robot motion in paragraph 4.2). Once $v$ is measured, the Doppler shift $f_{d}$ can be computed with (26), and the radar spectra can be shifted back in order to recover the correct radar-targets distances.

\subsection{Speckle filtering}

For a given radar-target distance $r$, the measured microwave signal can be seen as the sum of all the elementary reflectors or scatterers present in front of the radar at range $r$, so radar signal is subjected to fading effects [42],[43]. Indeed from (13), it can be seen that the sum is coherent: due the phase term $\Phi_{i}$ of the beat signal, the sum highlights constructive and destructive interferences. If the signals received from each reflector combine constructively, the resulting measured signal will highlight a high power level; but if the received signals combine destructively, the resulting signal will highlight a low or zero power. Under the assumptions that (i) the number of scatterers is large and scatterers are statistically identical and independent, and (ii) no single scatterer has a cross-section significantly larger than the average of all scatterers, the received power for each range bin will vary in a random fashion. If radar is of imaging type, these random amplitude variations produce a speckle pattern, which is a manifestation of fading statistics. An illustration of speckle noise can be seen in Figure 12.

Minimizing the effect of the speckle noise has been a major research activity, particularly in remote sensing domain. These methods can be non-adaptive such as median filter, or adaptive such as Lee or Frost filters [44]. Another technique used to mitigate speckle in radar remote sensing is multi-look 
filtering [45]. It is basically a non-coherent averaging of the radar image; it causes degradation in the image resolution, and it implies that the radar system offers a multi-look capability. As our radar is positioned on a moving platform, we can use the successive panoramic images to develop a specific multi-look filter. The principle of this approach is illustrated in Figure 17. The speckle reduction is obtained through a sliding non-coherent average of $P$ panoramic images. The $P$ panoramic images are projected onto a common space through a sliding dead reckoning phase: a gyrometer and an odometer measure the angular variations $\omega_{i}$ and the longitudinal displacements $\Delta_{i}$ between images, in order to compute their relative positions and orientations.

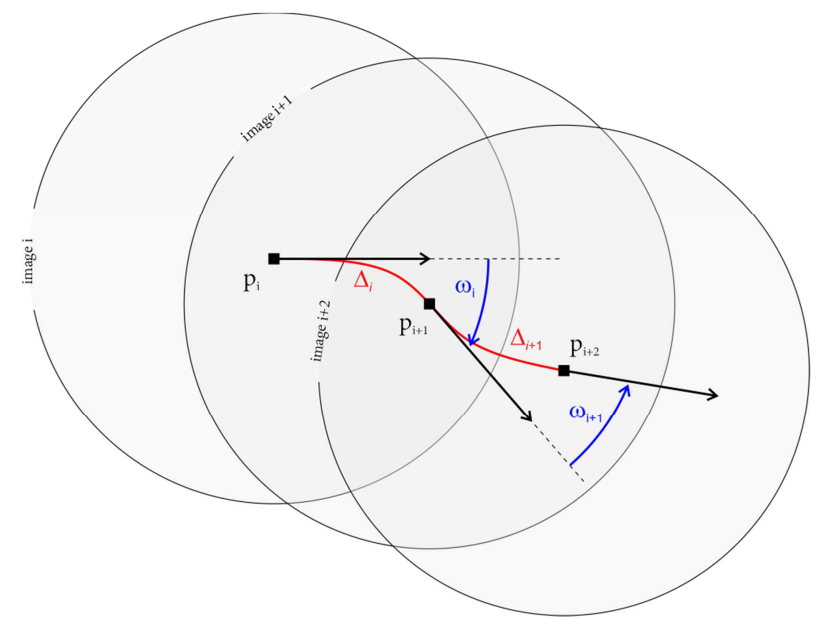

FIGURE 17: Principle of developed multi-look filter for Speckle filtering. A sliding dead reckoning phase computes the relative positions and orientations of $P$ panoramic radar images. Two proprioceptive sensors are used: a gyrometer to measure the angular variations $\omega_{i}$, an odometer to measure the longitudinal displacements $\Delta_{i}$. Once the $P$ images are positioned and oriented with respect to each other, a non-coherent average is computed.

A simulation of Speckle filtering is presented in Figure 18. The considered environment and radar configuration are described in Figure 11 and a raw image before filtering is shown in Figure 12. The robot velocity is $2 \mathrm{~m} / \mathrm{s}$, and $P=10$ successive images are used by the multi-look filter. This method allows reducing the speckle noise while preserving the resolution. The non-coherent average eliminates or strongly reduces the echoes from moving targets present in the environment, which is of minor important considering the static environment assumption imposed by the use of a sawtooth modulation. 


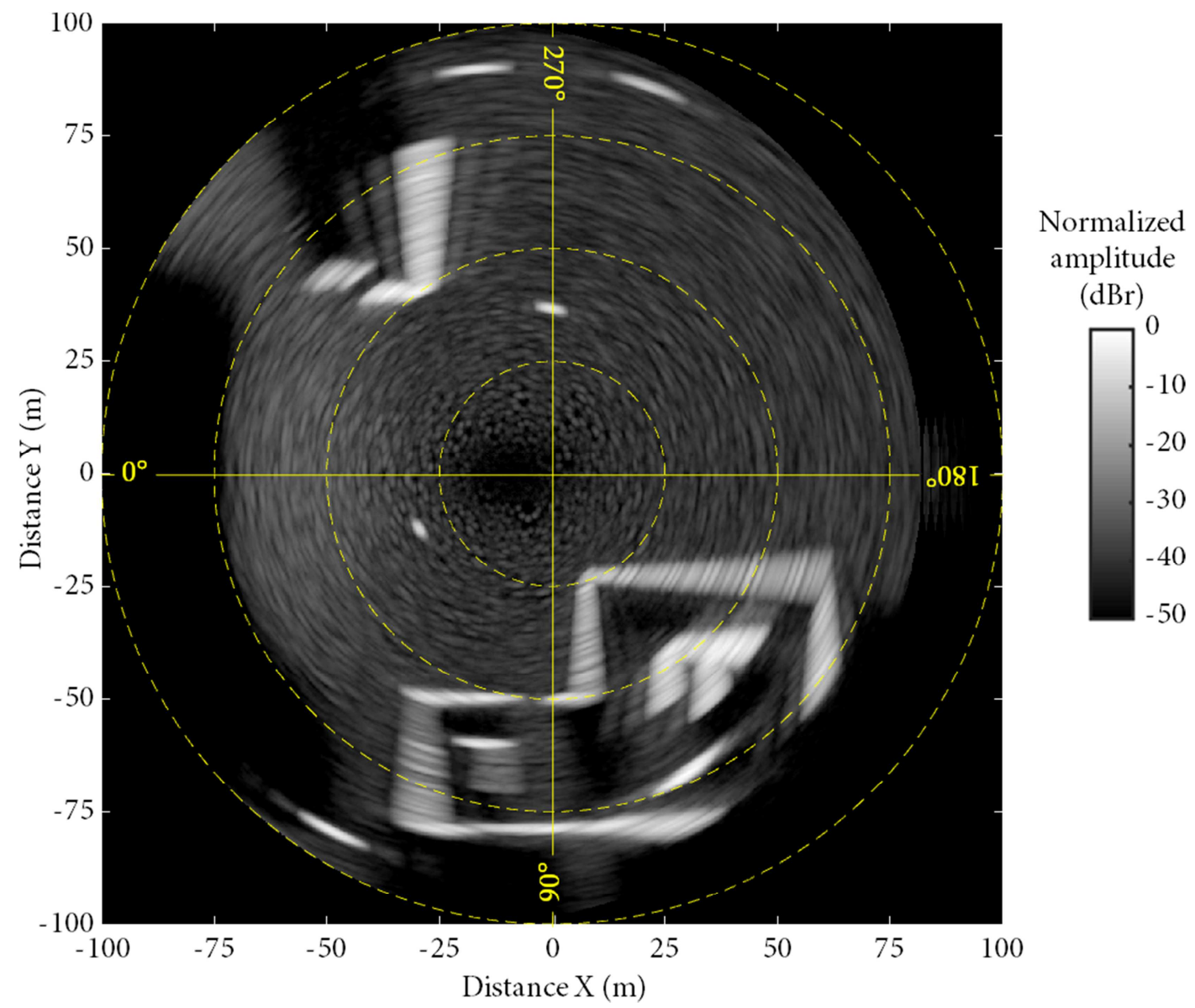

FIGURE 18: Example of Speckle noise filtering. The movement of the robot allows to obtain several images of the same zone from slightly different positions. In this example, the robot velocity is $2 \mathrm{~m} / \mathrm{s}$ and $P=10$ radar images are used by the multilook filter. The result of the filtering can be compared with the raw radar image in Figure 12(b).

\section{Conclusion}

PELICAN radar is a MMW radar designed for mobile robotics applications in outdoor environments. MMW radar highlights a low sensitivity to environment conditions such as weather events or dust, and it can provide a robust solution for perception in outdoor environments. PELICAN is based on FMCW principles, and it is using a rotating antenna in order to build radar images of the surrounding environment over $360^{\circ}$. This first of a two-part paper presented the theoretical basis of FMCW radars and of $2 \mathrm{D}$ images construction through simulation results. The interest of FMCW radars for shortrange applications is explained, as well as the limitations for simultaneous range and velocity measurements. The construction of $2 \mathrm{D}$ representations of the environment with signal measured with a fan-beam antenna is detailed. Specific corrections are made during the image construction process: robot motion corrections to take into account the combined effect of antenna rotation and robot movement; Doppler correction in order to eliminate the Doppler shift in radar spectra; and Speckle filtering in order to attenuate the grainy pattern in the radar images. The use of a sawtooth modulation with PELICAN radar offers a relative simplicity of hardware implementation, but it assumes a static environment condition. In the second part of the paper, the solutions adopted for the construction of PELICAN radar will be presented: hardware part (electronics, antenna, housing, etc.) and radar signal processing implementation. Experimental results obtained with several mobile platforms and various outdoor environments will be described. 


\section{Acknowledgments}

This study was supported by the Agence Nationale de la Recherche (ANR - the French national research agency) through the PELICAN research project (ANR-2010-EMMA-033). The PELICAN project was labeled by ViaMeca French pole of competitiveness. Researches were also supported by the French government research program "Investissements d'avenir" through the IMobS ${ }^{3}$ Laboratory of Excellence (ANR-10-LABX-16-01).

\section{Conflict of Interest}

The authors declare that there is no conflict of interest regarding the publication of this paper.

\section{References}

[1] L. Emmi, M. Gonzalez-de-Soto, G. Pajares, and P. Gonzalez-de-Santos, "New Trends in Robotics for Agriculture: Integration and Assessment of a Real Fleet of Robots," The Scientific World Journal, vol. 2014, Article ID 404059, 21 p., 2014.

[2] D.C. Slaughter, D.K. Giles, and D. Downey, "Autonomous robotic weed control systems: a review," Computers and Electronics in Agriculture, vol. 61, no. 1, pp. 63-78, 2008.

[3] Carré, ANATIS - Robot for hoeing and aid with decision-making, 2015, http://www.carre.fr/actualites/412anatis.html.

[4] Amazone, BoniRob field robot, 2009, http://info.amazone.de/DisplayInfo.aspx?id=14033.

[5] J. Hasch, E. Topak, R. Schnabel, T. Zwick, R. Weigel, and C. Waldschmidt, "Millimeter-Wave Technology for Automotive Radar Sensors in the $77 \mathrm{GHz}$ Frequency Band," IEEE Transactions on Microwave Theory and Techniques, vol.60, no. 3, pp. 845-860, 2012.

[6] M. Heuer, A. Al-Hamadi, A. Rain, and M.M. Meinecke, Detection and tracking approach using an automotive radar to increase active pedestrian safety, in Proceedings of the IEEE Intelligent Vehicles Symposium, pp. 890-893, Dearborn, USA, 8-11 June 2014.

[7] S. Heuel, and H. Rohling, "Pedestrian Classification in Automotive Radar Systems," in Proceedings of the $19^{\text {th }}$ International Radar Symposium, pp. 39-44, Warsaw, Poland, 23-25 May 2012.

[8] A. Hernandez-Gutierrez, J.I. Nieto, T. Bailey, and E.M. Nebot, "Probabilistic road geometry estimation using a millimeter-wave radar," in Proceedings of the IEEE International Conference on Intelligent Robots and Systems, 7 p., San Francisco, USA, 25-30 September 2011.

[9] G. Brooker, D. Jonhson, J. Underwood, J. Martinez, and L. Xuan, "Using the polarization of millimeterwave radar as a navigation aid," Journal of Field Robotics, vol. 32, no. 1, pp. 3-19, 2015.

[10] G. Brooker, R. Hennessey, M. Bishop, C. Lobsey, H. Durrant-Whyte and D. Birch, "High-resolution millimeter-wave radar systems for visualization of unstructured outdoor environments," Journal of Field Robotics, vol. 23, no. 10, pp. 891-912, 2006.

[11] G. Reina, A. Millela, and J. Underwood, "Self-learning classification of radar features for scene understanding," Robotics and Autonomous Systems, vol. 60, pp. 1377-1388, 2012.

[12] G. Reina, J. Underwood, G. Brooker, and H. Durrant-Whyte, "Radar-based perception for autonomous outdoor vehicles," Journal of Field Robotics, vol. 28, no. 6, pp. 894-913, 2011.

[13] A. Foessel-Bunting, J. Bares, and W. L. Whittaker, “Three-dimensional map building with MMW radar," in Proceedings of the International Conference on Field and Service Robotics, 6 p., Helsinki, Finland, June 2001.

[14] M. Adams, E. Jose, Robotic navigation and mapping with radar, Artech House, Boston, 2012.

[15] M. Jaud, R. Rouveure, L. Moiroux-Arvis, P. Faure, and M.O. Monod, "Boat borne radar mapping versus aerial photogrammetry and mobile laser scanning applied to river gorge monitoring," Open Journal of Remote Sensing and Positioning, vol. 1, pp. 48-63, 2014.

[16] T. Wang, N. Zheng, J. Xin, and Z. Ma, "Integrating Millimeter Wave Radar with a Monocular Vision Sensor for On-Road Obstacle Detection Applications,” Sensors, vol. 11, no. 9, pp. 8992-9008, 2011.

[17] G. El Natour, O. Ait-Aider, R. Rouveure, F. Berry, and P. Faure, “Toward 3D Reconstruction of Outdoor Scenes Using an MMW Radar and a Monocular Vision Sensor,” Sensors, vol. 15, no. 10, pp. 25937-25967, 2015. 
[18] G. Reina, A. Milella, and R. Rouveure, “Traversability Analysis for Off-Road Vehicles using Stereo and Radar Data," in Proceedings of the IEEE International Conference on Industrial Technology, 7 p., Seville, Spain, 17-19 March 2015.

[19] A. Millela, G. Reina, J. Underwood, and B. Douillard, "Visual ground segmentation by radar supervision," Robotics and Autonomous Systems, vol. 62, pp. 696-706, 2014.

[20] M.I. Skolnik, Introduction to radar systems, Electrical Engineering Series, McGraw-Hill International Editions, New-York, 2nd edition, 1980.

[21] D.K. Barton, Modern radar system analysis, Artech House, 1st edition, 1988.

[22] F.E. Nathanson, Radar design principles: signal processing and the environment, McGraw-Hill International Editions, New-York, 2nd edition, 1999.

[23] M.O. Monod, Frequency modulated radar: a new sensor for natural environment and mobile robotics, Ph.D. Thesis, Paris VI University, France, 1995.

[24] M.O. Monod, P. Faure, and R. Rouveure, "Intertwined linear frequency modulated radar and simulator for outdoor robotics applications," in Proceedings of the IEEE International Radar Conference (RADAR), 6 p., Bordeaux, France, 12-16 October 2009.

[25] A.W. Doerry, and B.C. Brock, "Radar cross section of triangular trihedral reflector with extended bottom plate," Sandia Report, Sandia National Laboratory, 2009.

[26] M.O. Monod, R. Chapuis, P. Gosset, R. Rouveure, D. Vivet, F. Gérossier, P. Faure, P. Checchin, L. Moiroux, P. Guérin, T. Humbert, and J. Morillon, "IMPALA project. Hyperfrequency radar for outdoor simultaneous localization and mapping," Traitement du Signal, vol. 29, no. 6, pp. 463-492, 2012.

[27] H. Rohling, and M.M. Meinecke, "Waveform design principles for automotive radar systems," in Proceedings of the CIE International Conference on Radar, pp. 1-4, Beijing, China, 15-18 October 2001.

[28] K.P. Corbley, “GeoSAR - Making Mapping the 'Impossible' Possible," GEO Informatics, vol. 13, no. 4, 2010.

[29] B.J. Allred, M.R. Ehsani, and J.J. Daniels, Handbook of Agricultural Geophysics, CRC Press LLC. Boca Raton, Florida, 2008.

[30] R. Rouveure, Short range soil characterization using microwaves techniques. Application to soil roughness measurement, Ph.D. Thesis, Clermont II University, France, 2001.

[31] S. Faruque, Radio Frequency Propagation Made Easy, SpringerBriefs in Electrical and Computer Engineering Series, Springer Editions, 2014.

[32] E.E. Altshuler, and R.A. Marr, "A comparison of experimental and theoretical values of atmospheric absorption at the longer millimeter wavelengths," IEEE Transactions on Antennas and Propagation, vol. 36, no. 10, pp. 1471-1480, 1988.

[33] A.J. Ansari, and B.G. Evans, "Microwave propagation in sand and dust storms," Communications, Radar and Signal Processing, IEE Proceedings F, vol. 129, pp. 315-322, 1982.

[34] Radio Regulations - Articles. Edition of 2012; International Telecommunication Union (ITU): Geneva, Switzerland, 2012.

[35] Tableau national de répartitions des bandes de fréquence. Edition de 2013. Agence Nationale des Fréquences, 2013.

[36] J.P. Ford, J.J. Plaut, C.M. Weitz, T.G. Farr, D.A. Senske, E.R. Stofan, G. Michaels, and T.J. Parker, Guide to Magellan Image Interpretation, Jet Propulsion Laboratory Publication: Pasadena, USA, 1993.

[37] A. Ferretti, A. Monti-Guarnieri, C. Prati, F. Rocca, and D. Massonnet, InSAR principles: guidelines for SAR interferometry processing and interpretation, Noordwijk, the Netherlands: ESA Publications Division, ESTEC, 2007.

[38] M.A. Sletten, G.O. Marmorino, and T.F. Donato, “An airborne, real aperture radar study of the Chesapeake Bay outflow plume,” Journal of Geophysical Research, vol. 104, no. C1, pp. 1211-1222, 1999.

[39] T.L. Ogle, W.D. Blair, R.J. Levin, and K.W. Harrigan, "Multiplatform-multisensor tracking with surveillance radars," in Proceedings of the 36th Southeastern Symposium on System Theory, pp. 190-194, Atlanta, USA, 2004.

[40] T.E. Wood, R.S. Ager, R.B. Fleury, and G.D. Heuer, Methods and apparatus for providing target altitude estimation in a two dimensional radar system, U.S. Patent 7417 583, Aug. 26, 2008.

[41] M. Jaud, R. Rouveure, P. Faure, M.O. Monod, and L. Moiroux-Arvis, "Correcting topography effects on terrestrial radar maps," in Proceedings of the IEEE International Radar Conference (RADAR), 6 p., Lille, France, 13-17 October 2014. 
[42] H. Mott., Remote Sensing with Polarimetric Radar - Appendix A: Fading and Speckle, John Wiley \& Sons, Inc., Hoboken, New Jersey, 2007.

[43] F. Argenti, A. Lapini, T. Bianchi, and L. Alparone, "A tutorial on speckle reduction in synthetic aperture radar images," IEEE Geoscience and Remote Sensing Magazine, vol. 1, no. 3, pp. 6-35, 2013.

[44] Y. Huang, and J.L. van Genderen, Evaluation of several Speckle filtering techniques for ERS-1\&2 imagery, in Proceedings of the International Society for Photogrammetry and Remote Sensing Congress, vol. XXXI, part. B, pp. 164-169, Vienna, Austria, 12-18 July 1996.

[45] A. Moreira, "Improved multilook techniques applied to SAR and SCANSAR imagery," IEEE Transactions on Geoscience and Remote Sensing, vol. 29, no. 4, pp. 529-534, 1991.
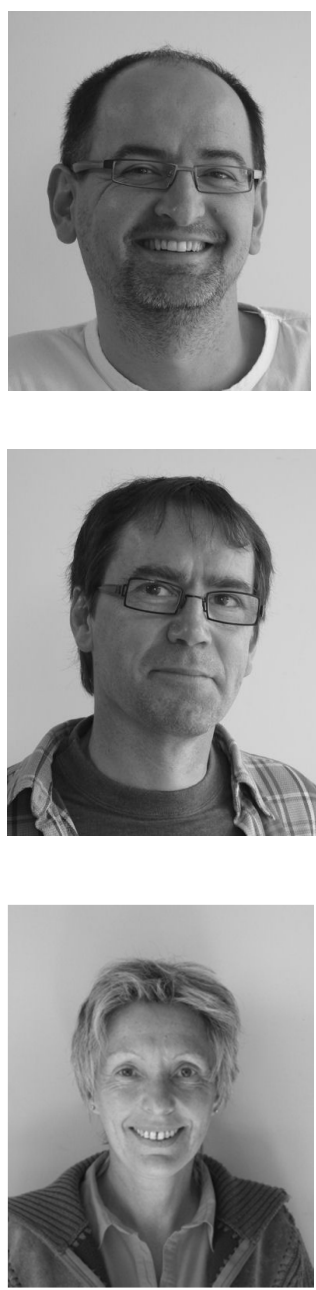

Raphaël Rouveure received the electrical engineering degree from the Polytech'Clermont School of Engineering, Clermont-Ferrand, France in 1990 and the Ph.D degree in Sciences For Engineer from the University of Clermont 2, France in 2001. He is associated with the Ecotechnologies department at the National Research Institute of Science and Technology for Environment and Agriculture (Irstea), France. His current research activities are in the development of new sensors for environmental application. His work deals with signal processing and technology in microwaves domain.

Patrice Faure received the D.E.A degree in Microelectronics from University of Montpellier, France in 1984. He is associated with the Ecotechnologies department at the National Research Institute of Science and Technology for Environment and Agriculture (Irstea), France. His research interests include the development of new sensors for environmental application. His work deals with signal processing and technology in microwaves and electronics domain.

Marie-Odile Monod received the electrical engineering degree from the Ecole Supérieure d'Ingénieurs en Electronique et Electrotechnique (ESIEE Group), Paris, France in 1977 and the Ph.D degree in Physics from the University of Paris VI, Paris, France in 1995. She is associated with the Ecotechnology and Agrosystem department at the CEMAGREF, France. Her research interests are in theoretical problems related to electromagnetic waves interaction with natural environment. She initiated activity in the radar sensors area for environmental perception and soil characterization. 